\title{
Analog Circuits and Signal Processing
}

Volume 128

\section{Series editors}

Mohammed Ismail, The Ohio State University, USA

Mohamad Sawan, École Polytechnique de Montréal, Canada 
More information about this series at http://www.springer.com/series/7381 
Valentijn De Smedt · Georges Gielen Wim Dehaene

\section{Temperature- and Supply Voltage-Independent Time References for Wireless Sensor Networks}

黛 Springer 
Valentijn De Smedt

Georges Gielen

Wim Dehaene

ESAT-MICAS

University of Leuven

Heverlee

Belgium

ISSN 1872-082X

ISBN 978-3-319-09002-3

DOI 10.1007/978-3-319-09003-0
ISSN 2197-1854 (electronic)

ISBN 978-3-319-09003-0 (eBook)

\section{Library of Congress Control Number: 2014945254 \\ Springer Cham Heidelberg New York Dordrecht London}

(C) Springer International Publishing Switzerland 2015

This work is subject to copyright. All rights are reserved by the Publisher, whether the whole or part of the material is concerned, specifically the rights of translation, reprinting, reuse of illustrations, recitation, broadcasting, reproduction on microfilms or in any other physical way, and transmission or information storage and retrieval, electronic adaptation, computer software, or by similar or dissimilar methodology now known or hereafter developed. Exempted from this legal reservation are brief excerpts in connection with reviews or scholarly analysis or material supplied specifically for the purpose of being entered and executed on a computer system, for exclusive use by the purchaser of the work. Duplication of this publication or parts thereof is permitted only under the provisions of the Copyright Law of the Publisher's location, in its current version, and permission for use must always be obtained from Springer. Permissions for use may be obtained through RightsLink at the Copyright Clearance Center. Violations are liable to prosecution under the respective Copyright Law. The use of general descriptive names, registered names, trademarks, service marks, etc. in this publication does not imply, even in the absence of a specific statement, that such names are exempt from the relevant protective laws and regulations and therefore free for general use.

While the advice and information in this book are believed to be true and accurate at the date of publication, neither the authors nor the editors nor the publisher can accept any legal responsibility for any errors or omissions that may be made. The publisher makes no warranty, express or implied, with respect to the material contained herein.

Printed on acid-free paper

Springer is part of Springer Science+Business Media (www.springer.com) 
The only reason for time is so that everything doesn't happen at once.

-A. Einstein 


\section{Preface}

"Most of what makes a book 'good' is that we are reading it at the right moment for us" is a quote from the Swiss-British philosopher Alain De Botton. The quote is right on top for a book where timing is the central given. Read this book about timing if at this moment your design is in need of an accurate yet efficient timing reference!

The book you are holding is the result of several years of Ph.D. research that started with the ambition to set important steps toward the implementation of batteryless wireless sensor nodes. Relatively soon it became clear that accurate timing is one of the key points in realizing this. Therefore the focus shifted to the construction of a low power, supply, and/or temperature independent timing reference. This topic in its turn surpasses the world of wireless sensor networks as it is much wider applicable.

We are convinced that the results we present here can help you in several ways. You can have a look at the circuits and concepts and adapt them for your system. More importantly the book can act as a source of inspiration for all those that are involved in sensor network design and the hardware for the Internet of Things. We dare to hope that the book brings you as much research inspiration as it brought us research joy while creating it.

Leuven, September 2014

Valentijn De Smedt

Georges Gielen

Wim Dehaene 


\section{Contents}

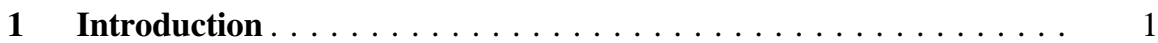

1.1 Historical Introduction. . . . . . . . . . . . . . 1

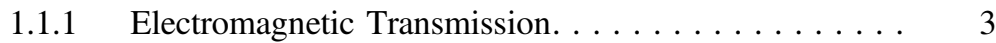

1.1.2 The Vacuum Tube ................. 8

1.1.3 The Invention of the Transistor. . . . . . . . . . 11

1.2 Wireless Sensor Networks . . . . . . . . . . . . . . . 17

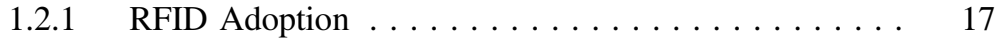

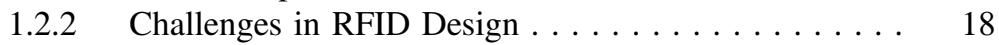

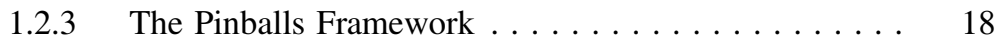

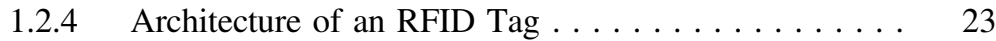

1.3 Focus and Outline of this Work ............. 26

\section{Part I Theoretical Background on Oscillators} and Time References

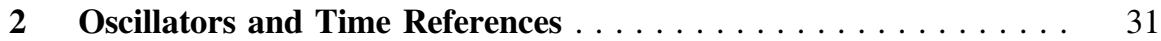

$2.1 \quad$ Introduction . . . . . . . . . . . . . . . 31

2.2 The Phase Space Description of an Oscillator......... 32

2.2.1 The Phase Space Description . . . . . . . . . . . . 32

2.2 .2 One-Dimensional Systems . . . . . . . . . . . . . 32

2.2 .3 Two-Dimensional Systems . . . . . . . . . . . . . . . 34

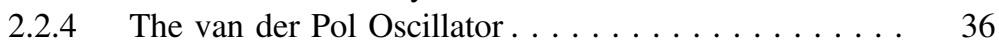

2.2.5 n-Dimensional Systems . . . . . . . . . . . . . . . . 39

2.3 Minimum Requirements for a Time Reference ......... 42

2.3.1 An Energy Reservoir and a Resistor . . . . . . . . 43

2.3.2 Two Different Energy Reservoirs ............. 44

2.3.3 Harmonic Versus Relaxation Oscillators ........ . 46 
2.4 Representation of an Oscillator Signal . . . . . . . . . . . . . . 47

2.4.1 Oscillator Signals in the Time Domain . . . . . . . . . 47

2.4.2 Oscillator Signals in the Frequency Domain....... . 49

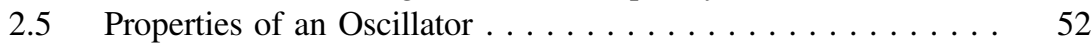

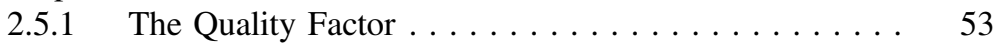

2.5.2 Stability of an Oscillator Signal . . . . . . . . . 59

2.6 Conclusion. . . . . . . . . . . . . . . . . . 59

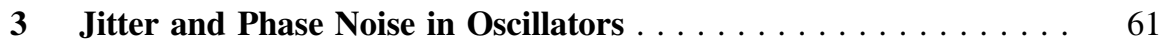

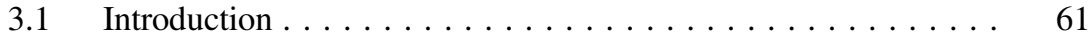

3.2 Noise Sources . . . . . . . . . . . . . . . . . . . . 62

$3.2 .1 \quad$ Noise in a Resistor . . . . . . . . . . . . . 63

3.2 .2 Noise in a P-N Junction. . . . . . . . . . . . . 63

3.2.3 MOS Transistor Noise. . . . . . . . . . . . . . . 64

3.3 The Phase Noise Spectrum. . . . . . . . . . . . . 64

3.3.1 The Noise Model of Leeson. . . . . . . . . . . . . . 65

3.4 The Phase Noise Theory of Hajimiri . . . . . . . . . . . . 68

3.4.1 Generation of the Phase Noise Spectrum . . . . . . . . 68

3.4.2 Extensions to the Theory of Hajimiri. . . . . . . . 74

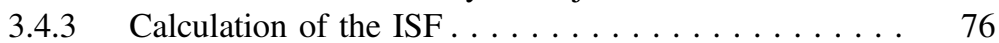

3.4.4 Evaluation of Hajimiri's Theory . . . . . . . . . 78

3.5 Nonlinear Noise Theories. . . . . . . . . . . . . . . . . . 79

3.5.1 The Lorentzian Spectrum. . . . . . . . . . . . . . . . . . . . . . . 79

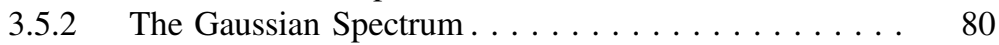

3.5 .3 Evaluation . . . . . . . . . . . . . . . 81

$3.6 \quad$ Phase Noise Versus Jitter. . . . . . . . . . . . . . . . . . . . . . . . 82

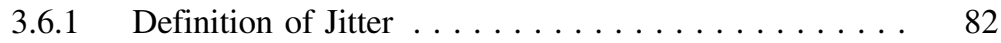

3.6.2 Only White Noise Sources. . . . . . . . . . . . 85

3.6.3 Colored Noise Sources . . . . . . . . . . . . . 85

3.6.4 General Calculation Method. . . . . . . . . . . . 86

3.7 The Q Factor and the Noise . . . . . . . . . . . . . . . 87

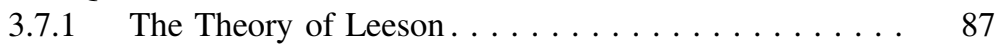

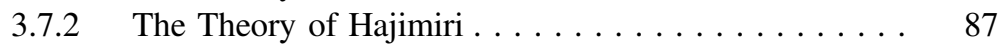

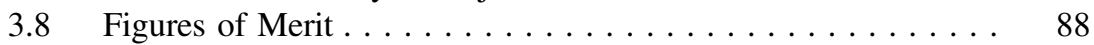

3.8 .1 The Phase Noise FoM. . . . . . . . . . . . 88

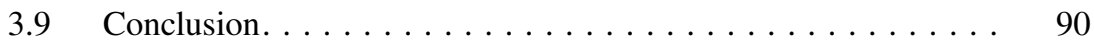

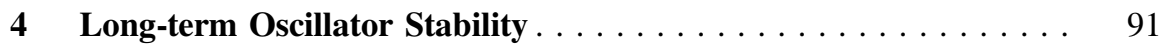

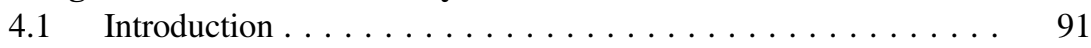

4.1.1 Causes of Frequency Drift . . . . . . . . . . . . . . . . . 91

4.1.2 Organization of this Chapter. . . . . . . . . . . 92

4.2 Building Blocks of an Oscillator. . . . . . . . . . 92

4.2.1 Linear Oscillator Systems . . . . . . . . . . 93 
4.2.2 Nonlinear Oscillator Systems . . . . . . . . . . . . . . . . 94

4.2.3 Transistor Behavior. . . . . . . . . . . . . . . . 100

4.2.4 Properties of the Feedback Network . . . . . . . . . . . 102

4.2.5 How to Obtain a Stable Oscillator? . . . . . . . . . . . . 106

4.3 Figures of Merit for Long-term Stability . . . . . . . . . . . . . 108

4.3.1 Temperature FoM . . . . . . . . . . . . . . . . . 108

4.3.2 Supply Voltage FoM. . . . . . . . . . . . . . . . . 109

4.4 Oscillators for Low-Power Applications. . . . . . . . . . . . . . . 110

4.4.1 Harmonic Integrated Oscillators . . . . . . . . . . . . . . . . 113

4.4.2 Relaxation Integrated Oscillators. . . . . . . . . . . . 121

$4.4 .3 \quad$ Ring Oscillators . . . . . . . . . . . . . . . . . . . . . . . . . . . . . 127

4.4 .4 Other Implementations. . . . . . . . . . . . . . . . . . . . . . 129

4.4.5 Comparison of the Different Topologies . . . . . . . 133

4.5 Conclusion. . . . . . . . . . . . . . . . . . 135

\section{Part II Oscillator Designs for Temperature} and Voltage Independence

5 Design of Two Wien Bridge Oscillators . . . . . . . . . . . . . . . . . . 139

5.1 Introduction . . . . . . . . . . . . . . . . . . . 139

5.1.1 The Wien Bridge Oscillator . . . . . . . . . . . . . . . 140

5.2 The Temperature-Independent Wien Bridge . . . . . . . . . . . 141

5.2.1 Basic Amplifier Structure. . . . . . . . . . . . . . . . . 142

5.2 .2 The Amplitude Regulator. . . . . . . . . . . . . . . 146

5.2 .3 Complete Circuit . . . . . . . . . . . . . . . . . . . . . 148

5.2.4 Phase Noise Performance. . . . . . . . . . . . . . . . . 148

5.2 .5 Measurement Results. . . . . . . . . . . . . 155

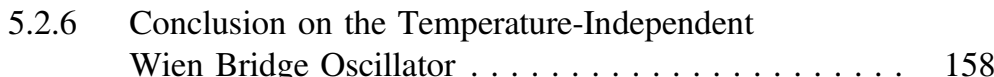

5.3 The Supply Voltage-Independent Wien Bridge Oscillator. . . . . 159

5.3 .1 The Oscillator Topology . . . . . . . . . . . . . . . . 160

5.3 .2 The Proposed Oscillator. . . . . . . . . . . . . 161

5.3 .3 The LDO Regulator . . . . . . . . . . . . . . . . . . 164

5.3.4 Temperature Dependency of the Voltage-Independent Oscillator. . . . . . . . . . . . . . . . 167

5.3.5 Measurement Results. . . . . . . . . . . . . . . . . 169

5.3.6 Conclusion on the Voltage-Independent Oscillator . . . 172

5.4 General Conclusion. . . . . . . . . . . . . . . . 172

6 The Pulsed Oscillator Topology . . . . . . . . . . . . . . . . . . . . . . 173

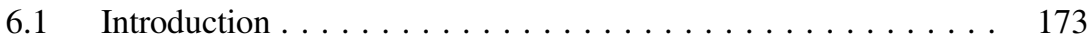

6.2 The Pulsed-Harmonic Oscillator Topology . . . . . . . . . . . . . 174

6.2.1 The Energy Tank . . . . . . . . . . . . . . 175 
6.3 Transient Behavior of the Energy Tank . . . . . . . . . . . 176

6.3.1 The n-th Order Transfer Function . . . . . . . . . . . . 177

6.3.2 Realistic Second-Order Tanks. . . . . . . . . . . . . . . . 179

6.4 Behavior of the Pulsed LC Oscillator . . . . . . . . . . . . . . 183

6.4 .1 Sensitivity to PW and MoI. . . . . . . . . . . . 186

6.4.2 Energy Losses During Oscillation . . . . . . . . . . . . . . 188

6.5 Phase Noise in the Pulsed LC Oscillator . . . . . . . . . . . . . . 189

6.5.1 Noise Injection During the Free-Running Period. . . . . 189

6.5.2 Noise Injection During the Applied Pulse . . . . . . . . 193

6.5.3 Impact of the Different Noise Sources . . . . . . . . . . . 194

6.6 Implementation of the Pulsed LC Oscillator . . . . . . . . . . . . . . . . . . 196

6.6.1 Design of the LC Tank . . . . . . . . . . . . . . 196

6.6.2 Design of the Differential Amplifier . . . . . . . . . . 198

6.6 .3 The Counter. . . . . . . . . . . . . . . . 200

6.6.4 The Pulse Generator . . . . . . . . . . . . . . . . 200

6.7 Measurement Results. . . . . . . . . . . . . . . . . . . 202

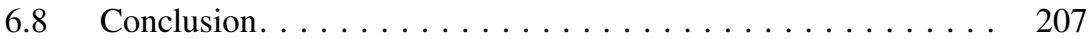

7 Injection-Locked Oscillators . . . . . . . . . . . . . . . . . . 209

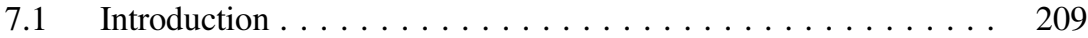

7.2 Injection Locking of an Oscillator. . . . . . . . . . . 211

7.2.1 Lock Range of the Oscillator . . . . . . . . . . . 211

7.2.2 Dynamic Behavior of the Locking Process. . . . . . . . 216

7.2 .3 Frequency Beating ................. 220

7.3 Phase Noise in the Injection-Locked Oscillator . . . . . . . . . . 222

7.3.1 Noise Model Using a Decreased Tank Impedance. . . . 223

7.3.2 A PLL-Based Noise Model . . . . . . . . . . . . . . . . 225

7.4 The Wirelessly-Locked Oscillator in $130 \mathrm{~nm} \ldots \ldots . . \ldots 229$

7.4.1 The Oscillator Topology . . . . . . . . . . . . . . . . 229

7.4.2 Techniques to Increase the Lock Range. . . . . . . . . . 233

7.4.3 Measurement Results. . . . . . . . . . . . . . 236

7.4.4 Conclusion on the 130-nm Injection-Locked

Oscillator. . . . . . . . . . . . . . . . . 237

7.5 The 40-nm Injection-Locked Receiver . . . . . . . . . . . . . . . . . . . . . . . . . . 238

7.5 .1 The Clock Circuit . . . . . . . . . . . . . . . . . . . 240

7.5.2 The Receiver Circuit . . . . . . . . . . . . . . . . 247

7.5.3 Measurement and Simulation Results . . . . . . . . 250

7.6 Conclusion. . . . . . . . . . . . . . . . . 256

8 Oscillator-Based Sensor Interfaces. . . . . . . . . . . . . . . . . . . 257

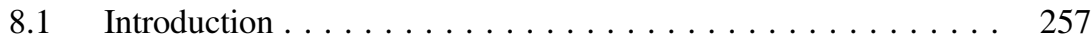

$8.2 \quad$ PLL-Based Sensor Interfaces . . . . . . . . . . . . . . . . . . . . . . 258

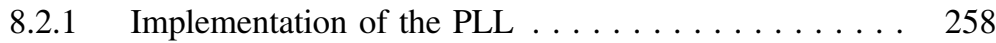


8.3 The PWM-Based Sensor Interface. . . . . . . . . . . . . . . . . 260

8.3.1 The Coupled Sawtooth Oscillator . . . . . . . . . . 261

8.3.2 Use in Combination with a Sensor . . . . . . . . . 263

8.3.3 Transmission of the Output Signal . . . . . . . . . . 265

8.4 Jitter in the Coupled Sawtooth Oscillator . . . . . . . . . . . . 266

8.4.1 Jitter due to Sensor Noise . . . . . . . . . . . . . . . 267

8.4.2 Jitter from the Differential Pair. . . . . . . . . . . . . 270

8.4 .3 Jitter due to the Current Source . . . . . . . . . . . 272

8.4.4 Noise Propagation to the Sensor Interface Output . . . 273

8.4.5 A/D-Converter FoM . . . . . . . . . . . . . . . 277

8.5 Implementation of the Sensor Interface . . . . . . . . . . . . . 278

8.5.1 Implementation in $130 \mathrm{~nm}$ CMOS . . . . . . . . . . 278

8.5.2 Implementation in $40 \mathrm{~nm}$ CMOS . . . . . . . . . . 282

8.5 .3 Measurement Results. . . . . . . . . . . . . . . . 288

8.6 Conclusion. . . . . . . . . . . . . . . . . . . . 292

\section{Part III Wireless Sensor Nodes}

9 Design of a Low-Power Wireless RFID Tag . . . . . . . . . . . . . . 295

9.1 Introduction . . . . . . . . . . . . . . . . . . . . . . . 295

9.2 Architecture of the Wireless Tag. . . . . . . . . . . . . . 296

9.2.1 The Clock and Receiver Circuit . . . . . . . . . . . . . 297

9.2.2 The UWB Transmitter. . . . . . . . . . . . . . . . . . 298

9.2 .3 The Sensor Interface . . . . . . . . . . . . . . . . . . . 299

9.2 .4 The Digital Logic . . . . . . . . . . . . . . . . . . . 300

9.3 Measurement Results. . . . . . . . . . . . . . . . . . . . 302

9.4 Conclusion. . . . . . . . . . . . . . . . . . . 305

10 Conclusion . . . . . . . . . . . . . . . . . . . . . . . . . . . . 307

10.1 Comparison to the State of the Art . . . . . . . . . . . 308

10.1.1 The Wien Bridge Implementations . . . . . . . . . . . . . . . 308

10.1.2 The Pulsed-Harmonic Oscillator . . . . . . . . . . . . 309

10.1.3 The Injection-Locked Oscillators. . . . . . . . . . 311

10.1 .4 The Sensor Interface . . . . . . . . . . . . . . . 311

10.1.5 The Wireless Tag .................... 312

10.1.6 General Conclusions . . . . . . . . . . . . . . . . . 312

10.2 Main Contributions . . . . . . . . . . . . . . . . . 313

10.3 Suggestions for Future Work . . . . . . . . . . . . . . 314 
Appendix A: Definitions and Conventions Used

Throughout the Work . . . . . . . . . . . . . . 317

Appendix B: Influence of a Nonlinear Amplifier . . . . . . . . . . . . . . 331

Appendix C: Measurement Issues for Jitter and Phase Noise. . . . . . . 341

Appendix D: Comparison to the State of the Art. . . . . . . . . . . . 353

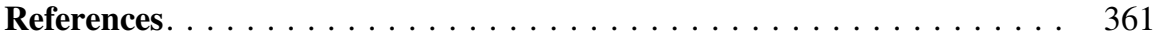

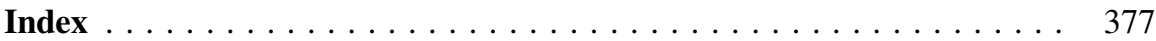




\section{Abstract}

Back in 2000, the Nobel prize committee recognized the invention of the integrated circuit in 1958 by Jack Kilby as one of the most far-reaching steps forward in modern technology. Today, almost 60 years after this invention, electronics are found everywhere in our society. This is mainly caused by the characteristic exponential growth factors in the electronics industry (Moore's law), which result in an exponential miniaturization and cost decrease. This evolution goes hand in hand with a similar growth of wireless communication technology: devices become smaller, frequencies and data rates higher. As a result, the wearability and functionality of wireless electronic devices drastically increase.

This ongoing technological progress is a direct cause of the appearance of Wireless Sensor Networks (WSN). An increasing number of autonomously operating devices is wirelessly connected to a network and/or to the Internet, an evolution which will eventually result in the so-called Internet of Things. Since both the miniaturization as well as the cost decrease of these wireless sensor nodes is necessary to become economically feasible, a growing need for fully-integrated, singlechip wireless devices is observed. The use of modern deep-submicron CMOS technologies in analog electronics, however, has several drawbacks in terms of temperature sensitivity and linearity.

This work elaborately investigates the possible circuit techniques to overcome the temperature and supply voltage sensitivity of fully integrated time references for ultra-low-power wireless communication in WSN. In a first step, the basic needs to build a frequency reference are studied. Furthermore, a closer look at the short-term as well as the long-term frequency stability of integrated oscillators is taken. This results in a design strategy, which is applied to six different oscillator design cases. All six implementations are subject to a study of phase noise and long-term frequency stability.

The first two implementations are respectively a temperature- and a supply voltage-independent Wien bridge oscillator. The temperature independence is obtained by using a novel feedback amplifier topology of which the output resistance only depends on a temperature stable resistor. This requires advanced circuit techniques and a highly-stable amplitude regulation circuit. The second Wien 
bridge implementation makes use of two nested regulators, resulting in an ultrahigh supply voltage stability over a wide voltage range.

The third design case makes use of a high-quality bondwire LC tank. A novel pulsed driving technique is developed to decrease the power consumption of the high-frequency oscillator circuit. This driving technique reduces the impact of the oscillator circuitry on the output frequency and therefore also on the temperatureand supply voltage stability of the oscillator. To better understand the application field of the pulsed oscillator topology, the noise performance is analyzed as well. The processed implementation is a unique combination of power consumption and long-term frequency stability.

Next, two injection-locked oscillator implementations are discussed. Apart from a stable output frequency, a high absolute accuracy is also obtained due to the locking to a wirelessly received RF signal. The first design uses the received $2.4 \mathrm{GHz}$ carrier frequency as a time reference. Despite its simple system topology, this approach has several drawbacks in terms of selectivity and power consumption. The second implementation locks to the envelope of the received RF signal. Therefore, the oscillator can run at a low frequency, drastically diminishing the power consumption. A second improvement is the addition of a network coordination receiver. For this purpose, a novel ultra-low-power receiver topology and demodulation technique are developed. As a result of the addressability, the overall power consumption in the network is reduced.

The last design case is a temperature- and supply voltage-independent oscillatorbased sensor interface. Since the challenge in this design is rather the stability of the output value than the frequency stability, a different design strategy is used. It is shown that the matching of different oscillator delay stages can be applied to obtain a stable and highly-linear digitalization of a sensor input.

The wirelessly injection-locked oscillator, the coordination receiver, the sensor interface, and a transmitter are combined into one highly-flexible wireless tag. The content, the scrambling code, and the length of the transmitted data burst can be adapted freely, depending on the application. The developed tag can therefore be used in a wide range of applications, with different accuracy requirements and energy constraints.

Finally, an elaborate comparison between the developed oscillator designs and the state of the art is performed. It is shown that the free-running implementations as well as the injection-locked designs improve the state of the art. This discussion results in several suggestions for possible future work. 


\section{About the Authors}

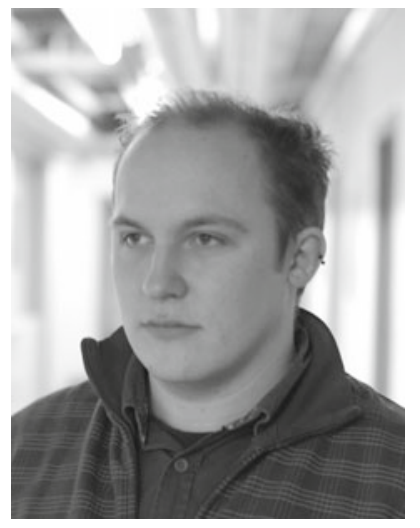

educational projects, some of them in co-operation with the IEEE Student Branch of Leuven.

$\mathrm{He}$ has been vice-chair technical activities of the IEEE student branch of Leuven between 2009 and 2013 and chaired the IEEE Student Branch and GOLD congress 2010 (SBC 2010). Since 2011, he is IEEE Benelux GOLD (Young Professionals) chair and co-chair of the IEEE SSCS Benelux chapter. Since 2009 he is a guest lecturer at ACE Group-T on UWB standards and Zigbee. 


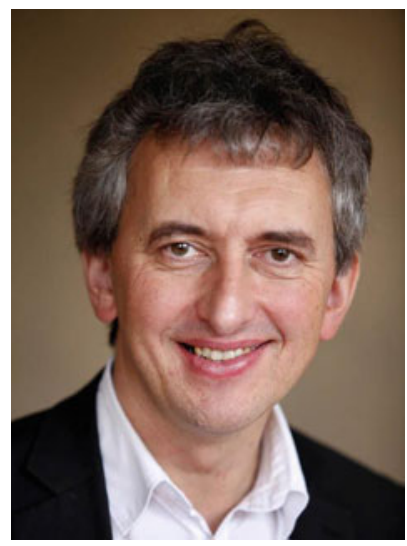

Georges G.E. Gielen received the M.Sc. and Ph.D. degrees in Electrical Engineering from the Katholieke Universiteit Leuven (KU Leuven), Belgium, in 1986 and 1990, respectively. He is a full professor at the Department of Electrical Engineering (ESAT). From August 2013, Georges Gielen is also appointed as vice-rector for the Group Science, Engineering and Technology and Academic Personnel of the KU Leuven.

His research interests are in the design of analog and mixed-signal integrated circuits, and especially in analog and mixed-signal CAD tools and design automation. He is a coordinator or partner of several (industrial) research projects in this area, including several European projects. He has authored or coauthored seven books and more than 450 papers in edited books, international journals, and conference proceedings. He is a Fellow of the IEEE since 2002.

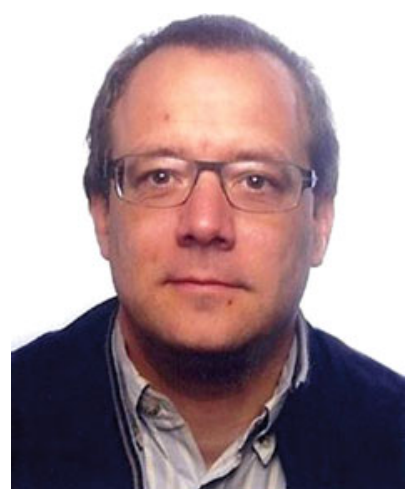

Wim Dehaene was born in Nijmegen, The Netherlands, in 1967. He received the M.Sc. degree in electrical and mechanical engineering in 1991 from the Katholieke Universiteit Leuven. In November 1996, he received the Ph.D. degree at the Katholieke Universiteit Leuven. His thesis is entitled "CMOS integrated circuits for analog signal processing in hard disk systems."

After receiving the M.Sc. Degree, Wim Dehaene was a research assistant at the ESAT-MICAS Laboratory of the Katholieke Universiteit Leuven. His research involved the design of novel CMOS building blocks for hard disk systems. The research was first sponsored by the IWONL (Belgian Institute for Science and Research in Industry and Agriculture) and later by the IWT (the Flemish institute for Scientific Research in the Industry). In November 1996, Wim Dehaene joined Alcatel Microelectronics, Belgium. There he was a senior project leader for the feasibility, design and development of mixed mode systems on chip. The application domains were telephony, xDSL, and high-speed wireless LAN. In July 2002, Wim Dehaene joined the staff of the ESAT-MICAS Laboratory of the Katholieke Universiteit Leuven where he is now a full professor. His research domain is circuit level design of digital circuits. The current focus is on ultra low power signal processing and memories in advanced CMOS technologies. Part of this research is performed in cooperation with IMEC, Belgium where he is also a part-time principal scientist. 
Wim Dehaene is teaching several classes on electrical engineering and digital circuit and system design. He is also very interested in the didactics of engineering. As such, he is guiding several projects aiming to bring engineering to youngsters and he is a teacher in the teacher education program of the KU Leuven.

Wim Dehaene is a senior member of the IEEE. Wim Dehaene is a member of the technical program committee of ESSCIRC and ISSCC. 


\section{Abbreviations}

A-ISF

AC

AM

BBPLL

BER

BJT

CMB

DAC

EEF

ELP

ENOB

ETF

FET

FLL

FoM

FRS

FSM

IFF

IoT

IR-UWB

ISF

JFET

LDO

LFSR

LTI

LTV

MEMS

MiM
Amplitude Impulse Sensitivity Function

Alternating Current

Amplitude Modulation

Bang-Bang Phase-Locked Loop

Bit Error Rate

Bipolar Junction Transistor

Cosmic Microwave Background

Digital-to-Analog Converter

Energy Enhancement Factor

Extremely-Low Power

Effective Number of Bits

Electro-Thermal Filter

Field-Effect Transistor

Frequency-Locked Loop

Figure of Merit

Fellow of the Royal Society

Finite State Machine

Identification Friend or Foe

Internet of Things

Impulse Radio Ultra-Wideband

Impulse Sensitivity Function

Junction Field-Effect Transistor

Low-DropOut

Linear Feedback Shift Register

Linear Time-Invariant

Linear Time-Variant

Microelectromechanical systems

Metal-insulator-Metal 


$\begin{array}{ll}\text { MoI } & \text { Moment of Impact } \\ \text { MoM } & \text { Metal-oxide-Metal } \\ \text { MOSFET } & \text { Metal-Oxide-Semiconductor Field-Effect Transistor } \\ \text { OCXO } & \text { Oven-Compensated Crystal Oscillator } \\ \text { P-UWB } & \text { Pulsed Ultra-Wideband } \\ \text { PC } & \text { Personal Computer } \\ \text { pcb } & \text { Printed Circuit Board } \\ \text { PDF } & \text { Probability Density Function } \\ \text { ppb } & \text { Parts per billion } \\ \text { ppm } & \text { Parts per million } \\ \text { PSD } & \text { Power Spectral Density } \\ \text { PVT } & \text { Process, Temperature and (Supply) Voltage } \\ \text { PW } & \text { Pulse Width } \\ \text { RF } & \text { Radio Frequency } \\ \text { RFID } & \text { Radio Frequency Identification } \\ \text { rms } & \text { Root mean square } \\ \text { SNDR } & \text { Signal to Noise and Distortion Ratio } \\ \text { SNR } & \text { Signal to Noise Ratio } \\ \text { TANSTAAFL } & \text { There Ain't No Such Thing As A Free Lunch } \\ \text { TCXO } & \text { Temperature-Compensated Crystal Oscillator } \\ \text { ULP } & \text { Ultra-Low Power } \\ \text { VCO } & \text { Voltage-Controlled Oscillator } \\ \text { VCXO } & \text { Voltage-Controlled Crystal Oscillator } \\ \text { WSN } & \text { Wireless Sensor Network } \\ \text { XO } & \text { Crystal Oscillator } \\ & \end{array}$




\section{Symbols}

$\begin{array}{ll}\alpha(\omega \cdot t) & \text { Deterministic, periodic function with period } 2 \pi \\ \alpha_{k} & \text { Phase shift of the } k \text {-th harmonic of a voltage oscillator } \\ & \text { signal } \\ \alpha_{\mu_{n}} & \text { Temperature coefficient of the electron mobility } \\ \alpha_{V_{t h}} & \text { Temperature coefficient of the transistor threshold voltage } \\ \beta & \text { Phase modulation index } \\ \beta_{k} & \text { Phase shift of the } k \text {-th harmonic of a voltage oscillator } \\ \Delta \omega_{3 d B} & \text { signal } \\ \Delta f_{-3 d B} & -3 \text { dB width of a resonant peak in a transfer function } \\ \Delta T_{n} & \text { Half-power width of an oscillator output spectrum } \\ \Delta V_{n} & \text { Delay error induced by noise } \\ \Delta V_{\text {rel }} & \text { Output voltage error induced by noise } \\ \mathscr{A}(\Delta \omega) & \text { Relative supply voltage span, as defined in Sect. } 4.3 .2 \\ \Delta \omega_{0} & \text { Amplitude noise density at frequency offset } \Delta \omega, \text { relative to } \\ & \text { the carrier } \\ \Delta \omega_{n} & \text { Difference between the fundamental frequency } \omega_{0} \text { and the } \\ & \text { natural frequency } \omega_{n} \text { of the tuned network } \\ \Delta \omega_{i n j} & \text { Angular frequency difference between the natural oscillator } \\ & \text { frequency } \omega_{n} \text { and the frequency of an injected signal } \omega_{i} \\ \Delta \omega_{n n} & \text { Frequency perturbation on the injected signal } i_{i n j}, \text { equal to } \\ & d \phi_{i n j}(t) / d t \\ & \text { Frequency perturbation on the free-running oscillator signal } \\ & \text { (not the injection-locked oscillator signal), equal to } \\ \Delta \phi_{i}(t) & d \phi_{n n}(t) / d t \\ & \text { Perturbation of the phase shift between an oscillator current } \\ \Delta \phi_{i n j}(t) & \text { and an injected current (due to noise), equal to } \\ \Delta \phi_{o s c} & \Delta \phi_{i n j}(t)-\Delta \phi_{o s c} \\ & \text { Perturbation on the phase of the injected signal } i_{i n j} \\ & \text { Perturbation on the phase of the (locked) oscillator signal }\end{array}$


$\delta_{k}$

$\delta_{X x}$

$\varepsilon$

$\varepsilon(t)$

$\gamma$

$\Gamma(x)$

$\Gamma_{i}(x)$

$\gamma_{k}$

$\Gamma_{\text {eff }}(x)$

$\Gamma_{r m s}$

$\Lambda(x)$

$\lambda_{i}$

$\Lambda_{i}(x)$

$\rho$

$\mu$

$\mu_{X x}$

$\Omega$

$\omega$

$\omega(t)$

$\omega_{0}$

$\omega_{B}$

$\omega_{i}$

$\omega_{L}$

$\omega_{m}$

$\omega_{n}$

$\Phi$

$\Phi(t)$

$\phi(t)$

$\phi_{\infty}$

Relative amplitude of the $k$-th harmonic of the voltage waveform, compared to the amplitude of the fundamental frequency

Skin-depth of a conducting material $X x$ (for instance Silver,

Ag)

Parameter equal to $A_{i n j} / A_{\text {osc }}$

Random amplitude variation of an oscillator signal

Constant parameter used to determine the bandwidth of a Lorentzian spectrum, equal to $\pi \cdot f_{0}^{2} \cdot c$

The Impulse Sensitivity Function (ISF) with period $2 \cdot \pi$

Impulse Sensitivity Function (ISF) on node $i$ with period $2 \cdot \pi$

Relative amplitude of the $k$-th harmonic of the voltage waveform, compared to the amplitude of the fundamental frequency

Effective Impulse Sensitivity Function (ISF), taking cyclostationary noise sources into account

Root mean square (rms) value of the Impulse Sensitivity Function (ISF)

Amplitude Impulse Sensitivity function (A-ISF), with period $2 \cdot \pi$

Eigenvalue with corresponding eigenvector $\mathbf{u}_{\mathbf{i}}$

Amplitude Impulse Sensitivity function (A-ISF) on node $i$, with period $2 \cdot \pi$

Generalized eigenvector with corresponding eigenvalue $\lambda$

Scalar nonlinearity parameter in the van der Pol equation

Magnetic permeability of a conducting material $X x$ (for instance Silver, $A g$ )

Unit of electrical resistance

Instantaneous angular frequency, pulsation, $2 \pi f$

Instantaneous angular frequency over time

Constant mean angular frequency of an oscillator, often used as the angular frequency in standard conditions

Beating frequency of an injected oscillator

Angular frequency of an injected signal

One-sided lock range of an injection-locked oscillator

Angular frequency of a motor

Natural angular frequency of an electrical (tuned) network, natural angular frequency of an oscillator, $2 \pi f_{n}$

magnetic flux due to the magnetic poles in a motor

Instantaneous phase of an oscillator signal

Random variations of noise in the phase function, also called excess phase function

Steady-state phase shift between the oscillator current and the injected current 


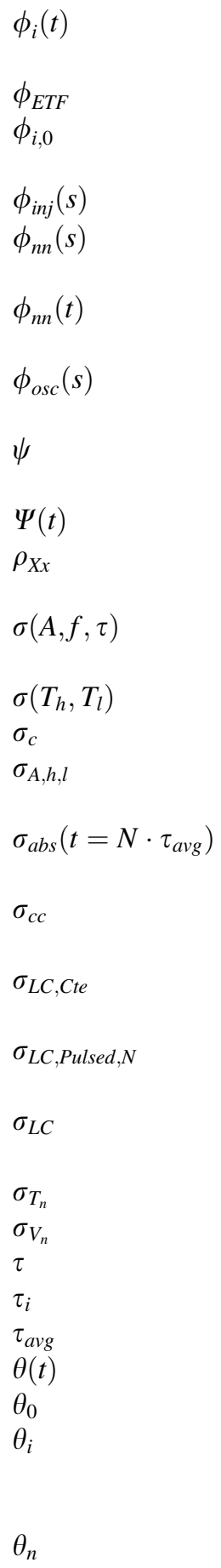

Phase shift between the oscillator current and the injected current Phase shift of an Electro-Thermal Filter Initial phase shift between the oscillator current and the injected current Phase shift of the injected signal in the frequency domain Phase shift of the free-running oscillator signal in the frequency domain

Phase shift of the free-running oscillator signal (not the injection-locked oscillator signal)

Phase shift of the locked oscillator signal in the frequency domain

Phase shift between the injected signal and the relaxation oscillator current

Systematic or deterministic variations in the phase function Conductivity of a conducting material $X x$ (for instance Silver, $A g$ )

Allan variance of the output of a function $f$ with dead time $\tau$ between the subsequent samples

Covariance between two stochastic periods

Cycle-to-cycle jitter of an oscillator output signal

Allan covariance between two variables $T_{h}$ and $T_{l}$ (with dead time $\tau$ )

Standard deviation of the absolute jitter after $N$ oscillation cycles, sometimes also called the absolute jitter

Alternative definition of the cycle-to-cycle jitter, calculated using the difference between subsequent periods

Average cycle-to-cycle jitter of the output waveform of a resonant tank with constant output amplitude

Standard deviation of the length of the pulsed period of an LC tank, normalized to $T_{L C} / 2$

Average cycle-to-cycle jitter of the output waveform of a resonant tank with decaying output amplitude

Standard deviation (rms) of the time noise on a stage delay Standard deviation (rms) of the voltage noise

An arbitrary moment in time

Period of the $i$-th oscillation cycle

Average period of an oscillator output signal

Phase shift of an oscillation over time (compared to $\omega_{n} \cdot t$ ) Initial phase shift of an oscillation

Phase shift induced by a small injected signal in the oscillator, angle between the oscillator signal and the resulting signal

Phase shift of a tuned network at $\omega \neq \omega_{n}$ 
$\Upsilon(t)$

A

$A_{0}$

$A_{1}$

A

$A_{D}$

$A_{d s}$

$A_{\text {env }, D C}$

$A_{\text {env }}(t)$

$A_{i}$

$A_{i n j}$

$A_{\text {osc }}$

$A_{R}$

$A_{r}$

$A(t)$

$A_{t}$

$A_{v}$

$B$

C

c

c

$C_{d s}$

$C_{g d}$

$C_{g s}$

$c_{i}$

$C_{o x}$

$C_{p}$

$C_{s}$

DC

$e$

$E_{\text {Decay }}(N)$

$E_{M}$

$E_{O s c}(N)$

$f(\Phi(t))$

F

$F_{\text {amp }}$

Deterministic and systematic amplitude variations of an oscillator signal

Voltage gain of a generic amplifier

Ideal constant amplitude of an oscillator signal

Linear gain of a voltage dependent transconductance amplifier

Ampère, unit of electrical current

Area or size of a diode junction

Amplitude of the drain-source current in an oscillator

DC approximation of the complex envelope of an oscillator output signal

Complex envelope of an oscillator output signal

$i$-th-order gain of a voltage dependent transconductance amplifier

Amplitude of a signal injected in an oscillator

Amplitude of an oscillator signal current

Area or size of a resistor

Amplitude of the resulting oscillator signal, sum of the injected current and the oscillator current

Instantaneous amplitude of an oscillator signal

Minimum attenuation of a feedback network

Voltage gain of an amplifier

Bandwidth of a modulating signal

Capacitance, a capacitor

Motor constant, speed of light

Constant parameter used to determine the bandwidth of a

Lorentzian spectrum

Drain-source capacitor of a MOS transistor

Gate-drain capacitor of a MOS transistor

Gate-source capacitor of a MOS transistor

Complex $i$-th pole frequency

Gate-oxide capacitance per unit area of a MOS transistor

Parallel capacitor in the equivalent quartz crystal model

Series capacitor in the equivalent quartz crystal model

Duty cycle of a digital signal

Euler's constant

Energy loss over $N$ oscillation cycles in a resonant tank with exponentially decaying output amplitude

Back electromotive force

Energy loss over $N$ oscillation cycles in a resonant tank with constant output amplitude

Periodic function of $\Phi(t)$ with period $2 \cdot \pi$

Device excess noise factor

Noise factor of an amplifier 


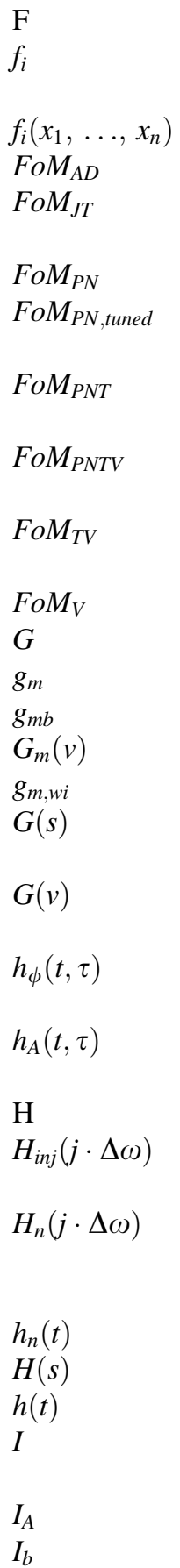

$\mathrm{F}$

FoM $_{A D}$

FoM $_{P N}$

${ }_{F o M} M_{P N, t u n e d}$

${ }_{F o M} M_{P N T}$

FoM $M_{P N T V}$

FoM $_{T V}$

FoM $_{V}$

$g_{m b}$

$g_{m, w i}$

$G(s)$

$G(v)$

$$
h_{\phi}(t, \tau)
$$$$
h_{A}(t, \tau)
$$

$\mathrm{H}$

$H_{i n j}(j \cdot \Delta \omega)$
Farad, unit of electrical capacitance

State variable of an oscillation on node $i$, normalized to amplitude and frequency

A smooth real-valued function of $x_{1}, \ldots, x_{n}$

Figure of Merit for Analog to Digital converters

Jitter and Temperature-dependency Figure of Merit (FoM) of an oscillator

Phase noise Figure of Merit (FoM) of an oscillator

Phase noise Figure of Merit (FoM) of an oscillator, taking the tuning sensitivity into account

Phase noise and Temperature-dependency Figure of Merit (FoM) of an oscillator

Phase noise, Temperature-dependency and Voltage-dependency Figure of Merit (FoM) of an oscillator

Temperature- and Voltage-dependency Figure of Merit (FoM) of an oscillator

Voltage-dependency Figure of Merit (FoM) of an oscillator Transconductance of a generic amplifier

Transconductance of a transistor

Transconductance of the bulk of a transistor

Gain of a nonlinear negative resistance or amplifier

Transconductance of a MOS transistor in weak-inversion

Transconductance of a generic amplifier, dependent on the Laplace variable (representing the frequency)

Input-amplitude-dependent transconductance of a generic amplifier

Unit impulse response for the excess phase for a charge injected at time $\tau$

Unit amplitude impulse response for a charge injected at time $\tau$

Henry, unit of electrical inductance

Transfer function of the phase perturbations on the injected signal $i_{i n j}$ to the injection-locked oscillator output

Transfer function of the phase perturbations on the freerunning oscillator signal $i_{\text {osc }}$ to the injection-locked oscillator output

Normalized impulse response of a resonant network

Transfer function of a linear system or network

Impulse response of a linear system or network

Unity matrix, symbol of electrical current, mechanical moment of inertia

Current amplitude through an inductor

Biasing current of an oscillator or an oscillator stage 


$I_{D 0}$
$i_{d, n}(t)$
$I_{d s}$
$i_{d s}$
$I_{d s, f}$
$I_{d s, w i}$
$i_{i n j}(t)$
$I_{i, r e g}$
$I_{i, r e g, r e p}$
$I_{k}$
$\Im(x)$
$I_{n}$
$i_{n 0}(t)$
$i_{n}(t)$
$i_{o s c}$
$i_{r}(t)$
$I_{(s)}$
$K_{i j}$
$K_{f T}(t)$
$j$
$j_{a b s}\left(t=N \cdot \tau_{a v g}\right)$
$J(d)$
$K_{D C}$
$J_{s}$
$k$
$k_{f R}$
$k_{30}$

Technology dependent parameter determining the weakinversion current of a MOS transistor Small-signal differential noise current Large-signal drain-source current of a MOS transistor Small-signal drain-source current of a MOS transistor Zero-temperature-coefficient DC biasing current of a transistor

Large-signal drain-source current of a MOS transistor in weak-inversion

Injected current in an oscillator

Regulated output current of a current regulation circuit

Regulated output current through the oscillator replica when the regulator is loaded with a resistor

Fourier coefficient of a current oscillator signal

The imaginary part of $x$

Amplitude of a noise current

Stationary current noise source

Injected small-signal noise current

Oscillator signal current

Resulting current in an oscillator, sum of the injected current and the oscillator current

Laplace transform of a current waveform

Small-signal current

Imaginary unit, $\sqrt{-1}$

Absolute jitter after $N$ oscillation cycles

Current density in a conductor, depending on the distance $d$ from the surface

Joule, unit of energy

Current density in a conductor at the surface

The Boltzmann constant, approximately equal to $1.38 \mathrm{~m}^{2} \cdot \mathrm{kg} \cdot \mathrm{s}^{-1} \cdot \mathrm{K}^{-1}$

Conversion gain of an envelope detector for an input signal with $100 \%$ modulation depth

Conversion gain of an envelope detector for an input signal with $30 \%$ modulation depth

Conversion gain of an envelope detector for a DC (lowfrequency) input signal

Technology dependent $1 / f$ noise fitting parameter of a diode

Technology dependent $1 / f$ noise fitting parameter of a resistor

Technology dependent $1 / f$ noise fitting parameter of a transistor

Residue of a partial fraction corresponding to real pole $i$ with $j$-th degree denominator 


\begin{tabular}{|c|c|}
\hline$K_{I L O}$ & Injection-locked oscillator gain \\
\hline$K_{m i x}$ & Mixing gain of a mixer circuit \\
\hline$K_{V C O}$ & $\begin{array}{l}\text { Integration constant or sensitivity of a } \mathrm{VCO} \text { to its control } \\
\text { voltage } v_{c}\end{array}$ \\
\hline$L$ & Inductance, an inductor \\
\hline$L$ & Length of a MOS transistor \\
\hline $\mathscr{L}(\Delta \omega)$ & $\begin{array}{l}\text { Phase noise density at frequency offset } \Delta \omega \text {, relative to the } \\
\text { carrier }\end{array}$ \\
\hline$L_{i j}$ & $\begin{array}{l}\text { Residue of a partial fraction corresponding to complex pole } \\
i \text { with } j \text {-th degree denominator }\end{array}$ \\
\hline $\mathscr{L}_{\text {total }}(\Delta \omega)$ & $\begin{array}{l}\text { Total noise power density at frequency offset } \Delta \omega \text {, relative } \\
\text { to the carrier }\end{array}$ \\
\hline MoI & Moment of Impact of a pulse applied to a resonant tank \\
\hline $\mathrm{MoI}_{N}$ & $\begin{array}{l}\text { Moment of Impact of a pulse applied to a resonant tank, } \\
\text { normalized to } T_{L C} / 2\end{array}$ \\
\hline$N$ & $\begin{array}{l}\text { Number of free-running periods in a Pulsed-Harmonic } \\
\text { oscillator }\end{array}$ \\
\hline$N F_{a m p}$ & Noise figure of an amplifier \\
\hline$N_{L F}$ & $\begin{array}{l}\text { Noise spectral density of the low-frequency noise at an } \\
\text { amplifier output (expressed in } \mathrm{V}^{2} / \mathrm{Hz} \text { ) }\end{array}$ \\
\hline$N_{o, E D}$ & $\begin{array}{l}\text { Noise spectral density of the noise at the output of an } \\
\text { envelope detector (expressed in } \mathrm{V}^{2} / \mathrm{Hz} \text { ) }\end{array}$ \\
\hline$N_{s r c}$ & $\begin{array}{l}\text { Noise spectral density of an input source (expressed in } \\
V^{2} / \mathrm{Hz} \text { ) }\end{array}$ \\
\hline$p$ & Frequency of a single pole \\
\hline$p_{i}$ & Real $i$-th pole frequency \\
\hline$P(s)$ & Numerator of a transfer function $H(s)$ \\
\hline$P_{s}$ & Average power dissipated in a resonant tank \\
\hline$P_{s b c}(\Delta \omega)$ & $\begin{array}{l}\text { Single-sideband noise power at offset frequency } \Delta \omega \text {, } \\
\text { relative to the carrier }\end{array}$ \\
\hline $\mathscr{P}_{\text {side }}\left(f_{0}+\Delta f, 1 \mathrm{~Hz}\right)$ & $\begin{array}{l}\text { Single-sideband noise power in a } 1 \mathrm{~Hz} \text { interval at a } \\
\text { frequency offset } \Delta f \text { from the carrier }\end{array}$ \\
\hline$P W$ & Pulse width of a pulse applied to a resonant tank \\
\hline$P W_{N}$ & $\begin{array}{l}\text { Pulse width of a pulse applied to a resonant tank, } \\
\text { normalized to } T_{L C} / 2\end{array}$ \\
\hline$Q$ & Quality factor of a resonant (second order) network \\
\hline$q$ & Unit charge of a single charge carrier (electron or hole) \\
\hline$Q_{C}$ & Quality factor of a capacitor $C$ \\
\hline$q_{\text {Cte }}$ & $\begin{array}{l}\text { Maximum charge displacement (compared to equilibrium) } \\
\text { in a resonant network with constant output amplitude } \\
\text { during one period }\end{array}$ \\
\hline$q_{\text {Decay }}$ & $\begin{array}{l}\text { Maximum charge displacement (compared to equilibrium) } \\
\text { in a resonant network with exponentially decaying output } \\
\text { amplitude during one period }\end{array}$ \\
\hline
\end{tabular}




\begin{tabular}{|c|c|}
\hline$Q_{G}$ & Generalized Q factor \\
\hline$Q_{L}$ & Quality factor of an inductor $L$ \\
\hline$q_{\max }$ & $\begin{array}{l}\text { Maximum charge displacement from the equilibrium state } \\
\text { on the output node of a resonant tank during one oscillation } \\
\text { cycle }\end{array}$ \\
\hline$Q(s)$ & Denominator of a transfer function $H(s)$ \\
\hline$R$ & Resistance, a resistor \\
\hline$r_{0}$ & $\begin{array}{l}\text { Small signal output (drain) impedance of a MOS transistor } \\
\text { (also called } r_{d s} \text { ) }\end{array}$ \\
\hline$R_{B}$ & Bulk resistance of a transistor \\
\hline$R_{D}$ & Drain resistance of a transistor \\
\hline$r_{d s}$ & $\begin{array}{l}\text { Small signal output (drain) impedance of a MOS transistor } \\
\text { (also called } r_{0} \text { ) }\end{array}$ \\
\hline$R_{\text {eff }}$ & Effective noise resistance of a transistor \\
\hline$\Re(x)$ & The real part of $x$ \\
\hline$R_{G}$ & Gate resistance of a transistor \\
\hline$R_{p}$ & Equivalent parallel resistance of an RLC network \\
\hline$R_{S}$ & Source resistance of a transistor \\
\hline$R_{S}$ & Series resistance of an inductor \\
\hline$s$ & $\begin{array}{l}\text { Laplace variable, equal to } \sigma+j \cdot \omega \text {, in steady-state equal to } \\
j \cdot \omega\end{array}$ \\
\hline $\mathrm{S}$ & Second, unit of time \\
\hline$S_{\phi, f}(f)$ & $\begin{array}{l}\text { Power Spectral Density of the phase fluctuations as a result } \\
\text { of the } 1 / f \text { noise component }\end{array}$ \\
\hline$S_{a}(f)$ & Power Spectral Density of a waveform $a$ \\
\hline$S_{i_{n}}(f)$ & Power Spectral Density of a current noise source $i_{n}$ \\
\hline$S_{i n j}(\omega)$ & Power spectral density of the injected signal $i_{i n j}$ \\
\hline$S_{n n}(\omega)$ & Power spectral density of the free-running oscillator signal \\
\hline$S_{\text {osc }}(\omega)$ & Power spectral density of the locked oscillator signal $i_{o s c}$ \\
\hline$S_{R F}(f)$ & Power Spectral Density of a general RF signal \\
\hline$S_{V_{n}}(f)$ & Power Spectral Density of the voltage noise on a capacitor \\
\hline$S_{v_{n}}(f)$ & Power Spectral Density of a voltage noise source $v_{n}$ \\
\hline$S_{x}(f)$ & Power Spectral Density of phase time fluctuations \\
\hline$S_{y}(f)$ & Power Spectral Density of fractional frequency fluctuations \\
\hline$S_{\phi}(f)$ & Power Spectral Density of phase fluctuations \\
\hline$S_{\Delta f}(f)$ & Power Spectral Density of frequency fluctuations \\
\hline$S_{\Delta \omega}(f)$ & Power Spectral Density of angular frequency fluctuations \\
\hline$T$ & $\begin{array}{l}\text { Absolute temperature, expressed in Kelvin }[\mathrm{K}] \text { or (depend- } \\
\text { ing on the context), the oscillation period }\end{array}$ \\
\hline$t$ & Time variable \\
\hline$T_{0}$ & Period of an oscillator \\
\hline$T_{D}$ & Delay of an oscillator stage \\
\hline$t_{d}$ & Delay of an inverter \\
\hline
\end{tabular}




\begin{tabular}{|c|c|}
\hline$T_{h}$ & $\begin{array}{l}\text { Time span that a digital oscillator output is high during one } \\
\text { period }\end{array}$ \\
\hline$T_{h, 0}$ & $\begin{array}{l}\text { Time span that a digital oscillator output is high during one } \\
\text { period with a sensor input signal equal to zero }\end{array}$ \\
\hline$t_{h l}$ & $\begin{array}{l}\text { Input-output delay of an amplifier for a falling edge at the } \\
\text { output }\end{array}$ \\
\hline$T_{i, r e g}$ & Loopgain of a current regulation circuit \\
\hline$T_{l}$ & $\begin{array}{l}\text { Time span that a digital oscillator output is low during one } \\
\text { period }\end{array}$ \\
\hline$t_{L}$ & $\begin{array}{l}\text { Time needed for an injection-locked oscillator to lock, lock } \\
\text { time of an injection-locked oscillator }\end{array}$ \\
\hline$T_{l, 0}$ & $\begin{array}{l}\text { Time span that a digital oscillator output is low during one } \\
\text { period with a sensor input signal equal to zero }\end{array}$ \\
\hline$T_{L C}$ & Period of a free-running LC tank \\
\hline$t_{l h}$ & $\begin{array}{l}\text { Input-output delay of an amplifier for a rising edge at the } \\
\text { output }\end{array}$ \\
\hline$T_{M}$ & Motor torque \\
\hline$T_{O s c}$ & Complete period of a multi-stage oscillator \\
\hline$T(s)$ & Frequency dependent loopgain of a feedback system \\
\hline$T_{\text {Sens }}$ & Sensitivity of the output frequency $f_{0}$ to temperature \\
\hline$T_{\text {Switch }}$ & $\begin{array}{l}\text { Time interval needed for a differential oscillator stage to } \\
\text { switch }\end{array}$ \\
\hline$T(t)$ & Phase time \\
\hline$T_{v, \text { reg }}$ & Loopgain of a voltage regulation circuit \\
\hline$U$ & Symbol of electrical tension \\
\hline $\mathbf{u}_{\mathbf{i}}$ & Eigenvector with corresponding eigenvalue $\lambda_{i}$ \\
\hline$u(t)$ & The Heaviside function \\
\hline$V_{A}$ & Voltage amplitude over a capacitor \\
\hline$V_{b s}$ & Large-signal bulk-source voltage of a MOS transistor \\
\hline$v_{b s}$ & Small-signal bulk-source voltage of a MOS transistor \\
\hline$v_{c}(t)$ & Control voltage of a VCO \\
\hline$V_{C t e}$ & Constant input voltage \\
\hline$V_{c t r l}$ & Control voltage to control the gain of an amplifier \\
\hline$V_{d}$ & Differential voltage applied to the input of a differential pair \\
\hline$V_{d d}$ & Supply voltage \\
\hline$V_{d s}$ & Large-signal drain-source voltage of a MOS transistor \\
\hline$v_{d s}$ & Small-signal drain-source voltage of a MOS transistor \\
\hline$V_{E}$ & Early-voltage of a MOS transistor \\
\hline$V_{g s}$ & Large-signal gate-source voltage of a MOS transistor \\
\hline$v_{g s}$ & Small-signal gate-source voltage of a MOS transistor \\
\hline$\hat{V}_{g s, f}$ & $\begin{array}{l}\text { Zero-temperature-coefficient DC biasing voltage of a } \\
\text { transistor }\end{array}$ \\
\hline$V_{g t}$ & Overdrive voltage of a MOS transistor, equal to $V_{g s}-V_{t h}$ \\
\hline$V_{i}$ & Output voltage of the $i$-th oscillator stage \\
\hline
\end{tabular}




\begin{tabular}{|c|c|}
\hline$V_{i, \text { reg }}$ & Regulated output voltage of a current regulation circuit \\
\hline$V_{k}$ & Fourier coefficient of a voltage oscillator signal \\
\hline$V_{\max }$ & $\begin{array}{l}\text { Maximum supply voltage at which a circuit can properly } \\
\text { operate }\end{array}$ \\
\hline$v_{\max }$ & $\begin{array}{l}\text { Maximum output voltage (compared to equilibrium) of a } \\
\text { resonant tank during one oscillation cycle }\end{array}$ \\
\hline$V_{\text {min }}$ & $\begin{array}{l}\text { Minimum supply voltage at which a circuit can properly } \\
\text { operate }\end{array}$ \\
\hline$v_{n, \text { diff }}$ & Differential noise voltage \\
\hline$v_{n}(t)$ & Small-signal noise voltage \\
\hline$V_{r e f}$ & Output voltage of a voltage reference \\
\hline$V_{\text {reg }}$ & Output voltage of a voltage regulator \\
\hline$V_{\text {rep }}$ & Output biasing voltage coming from a replica circuit \\
\hline$V(s)$ & Laplace transform of a voltage waveform \\
\hline$V_{S}$ & $\begin{array}{l}\text { Output voltage of a sensor, input voltage of a sensor } \\
\text { interface }\end{array}$ \\
\hline$V_{S, D C}$ & DC value of the sensor output voltage \\
\hline$V_{\text {Sens }}$ & Sensitivity of the output frequency $f_{0}$ to the supply voltage \\
\hline$V_{S_{-}}$ & Negative voltage output of a differential sensor \\
\hline$V_{S+}$ & Positive voltage output of a differential sensor \\
\hline$v(t)$ & Small-signal voltage \\
\hline$v(t-\tau)$ & Decay function of the excess amplitude \\
\hline$V_{T}$ & Threshold voltage of a relaxation oscillator \\
\hline$V_{t}$ & $\begin{array}{l}\text { Thermal voltage, equal to } k \cdot T / q=26 \mathrm{mV} \text { at room } \\
\text { temperature }\end{array}$ \\
\hline$V_{t h}$ & Threshold voltage of a MOS transistor \\
\hline V & Volt, unit of electrical tension \\
\hline$V_{v, r e g}$ & Regulated output voltage of a voltage regulation circuit \\
\hline$V_{v, \text { reg,rep }}$ & $\begin{array}{l}\text { Regulated output voltage over the oscillator replica when } \\
\text { the regulator is loaded with a resistor }\end{array}$ \\
\hline$V_{v, \text { reg, res }}$ & $\begin{array}{l}\text { Regulated output voltage of a voltage regulation circuit } \\
\text { loaded with a resistor }\end{array}$ \\
\hline$W$ & Width of a MOS transistor \\
\hline$x$ & $\begin{array}{l}\text { Normalized variable of the ISF } \Gamma(x) \text {, typically equal to } \\
\omega_{n} \cdot t\end{array}$ \\
\hline$\dot{x}_{i}$ & Time-derivative of $x_{i}$ \\
\hline$x_{i}(t)$ & A real-valued function of $t$ \\
\hline$x(t)$ & Random instantaneous phase time variation, $\phi(t) / \omega_{0}$ \\
\hline$y(t)$ & Instantaneous fractional frequency variation, $d x(t) / d t$ \\
\hline$Z$ & Complex impedance \\
\hline $\mathrm{Z}$ & Scalar for which $z \in \mathbb{Z}$ \\
\hline$Z_{k}$ & $\begin{array}{l}\text { Impedance of a resonant network, seen by the } k \text {-th } \\
\text { harmonic }\end{array}$ \\
\hline
\end{tabular}




\section{Figures}

Fig. 1.1 a Schematic drawing of The Thing. The working principle is based on the reflection of an RF signal by the antenna. The load impedance of the antenna is changing as a function of the sound pressure. $\mathbf{b}$ The Thing was hidden in a replica of the Great Seal [292] .................... 2

Fig. 1.2 a Schematic drawing on the electromagnetic rotation experiment or homopolar motor. b Schematic drawing of an induction experiment [304].

Fig. 1.3 James Clerk Maxwell proved mathematically that the rings of Saturn consist of numerous small particles [315].

Fig. 1.4 Nikola Tesla aged 36 [291] and two of his patents, one for the efficient generation of RF power, a second for his Tesla

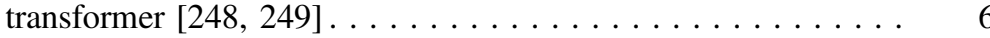

Fig. 1.5 a Photograph of Marconi's Magnetic Detector. b Schematic drawing of the Magnetic Detector $[237,302] \ldots \ldots \ldots \ldots$

Fig. 1.6 Photograph of a crystal detector or cat's whisker. The metalsemiconductor junction which was created is nowadays known as a Schottky diode [287] . . . . . . . . . . . 8

Fig. 1.7 The RMS Titanic was believed to be unsinkable [308] . . . . . 9

Fig. 1.8 Schematic of the autodyne or regenerative receiver. The photo on the right shows the simplicity of this circuit [307] . .

Fig. 1.9 Idealized cross-section of an $n$-channel (left) and $p$-channel (right) JFET. By reversely biasing the $p n$ junction at the gate, the conducting channel is pinched off [97] . . . . . . . 11

Fig. 1.10 Cross section of a typical npn (left) and pnp (right) transistor structure. . . . . . . . . . . . . . . . . .

Fig. 1.11 Cross-section of an NMOS (left) and PMOS (right) transistor. Even in unbiased condition a depletion region is present surrounding the source and drain doping implant ... 
Fig. 1.12 One of the illustrations of Kilby's patent No. 3261081. This device is considered to be the first integrated circuit [130] . . .

Fig. 1.13 Logarithmic plot of the number of calculations per second per $\$ 1,000$. From the early beginning, this curve increases

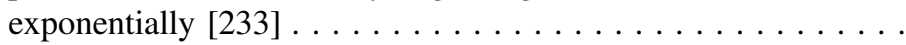

Fig. 1.14 System overview of the three hierarchical layers in the Pinballs platform. . . . . . . . . . . . . . .

Fig. 1.15 Typical charge and discharge characteristic of the supply curve on an RFID tag. Most often the scavenging mechanism is rather slow, which results in a $T_{\text {charge }}$ in the order of minutes. Depending on the load current and the size of the capacitor, $T_{a c t}$ typically varies between $1 \mathrm{~ms}$ and several seconds. The higher $V_{\max }$ and the lower $V_{\min }$, the longer the tag circuitry can be active without interruptions (one burst

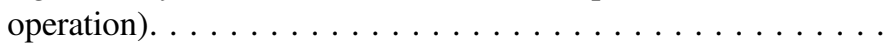

Fig. 1.16 Architecture of a wireless RF-powered tag . . . . . . . . . . 23

Fig. 2.1 The graphical representation of the 1-dimensional phase space gives a good insight in the first-order system's behavior. All the possible states of the system are represented by unique points on the horizontal axis. The vertical axis is only added for better understanding. . . . . . . . . .

Fig. 2.2 Phase portraits of different linear second-order systems with a two positive eigenvalues, $\mathbf{b}$ a positive and a negative eigenvalue, $\mathbf{c}$ two negative eigenvalues. $\mathbf{a}$ and $\mathbf{b}$ are both called unstable systems, $\mathbf{c}$ is a stable system...........

Fig. 2.3 Phase portraits of different linear second-order systems with two complex conjugate eigenvalues. The real part has a a positive sign, $\mathbf{b}$ a value equal to zero, $\mathbf{c}$ a negative real part. $\mathbf{a}$ is called an unstable system, $\mathbf{b}$ is marginally stable and $\mathbf{c}$ is a stable system . . . . . . . . . . . . . . . .

Fig. 2.4 Phase portraits and output signal of the van der Pol oscillator for different values of $\mu$. The initial value for the blue curve is $(0.01,0.01)$, for the green curve this is $(3,3)$. On each phase portrait the 50 blue circles are equidistant in time to show the behavior of the oscillator during the limit cycle. a $\mu=0.1, \mathbf{b} \mu=0.5, \mathbf{c} \mu=5, \mathbf{d} \mu=20 \ldots \ldots \ldots \ldots$

Fig. 2.5 Behavior of the van der Pol oscillator with a PI amplitude regulator, $A=1 / \sqrt{2}, \mu=0.05$ and $\eta=0.01$. a Shows the behavior in the time domain. The output signal $x_{1}(t)$ is shown on the left. On the right $x_{3}(t)$ is plotted, which corresponds to the resulting output signal of the amplitude regulator. b Shows the behavior in the phase plane/space; the overshoot of the amplitude is clearly visible. It takes a lot of periods before the limit cycle (on the right) is reached...... 
Fig. 2.6 Chaotic behavior of the forced modified van der Pol equation. The circles show the evolution of 32 near start conditions over time. The blue line is an $x_{1}-x_{2}$ plot of one

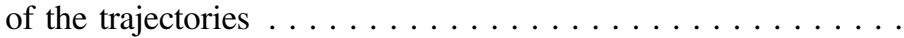

Fig. 2.7 Two coupled energy tanks, an inductor $L$ and a capacitor $C$, forming an oscillator ................

Fig. 2.8 An inductor can exchange its energy with a kinetic energy tank. A DC motor is needed to do the energy conversion. . . . .

Fig. 2.9 Different RLC tanks. The left tank (a) is not driven and its losses are represented by the parallel resistor. In (b) the same tank is driven by a transconductance amplifier. In (c) the losses are represented by a series resistor in the inductor, which is the closest to the real situation . . . . . . . . . .

Fig. 2.10 Different definitions of the $\mathrm{Q}$ factor of a two-pole system, based on the width (bandwidth) of the resonant peak and based on the steepness of the phase shift of the feedback

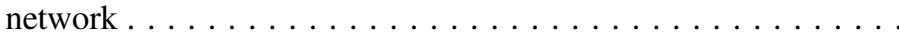

Fig. 3.1 Phase noise spectrum at the output of a generic oscillator. According to the power-law noise model, the spectrum can be divided in different zones, with a different noise origin or underlying mechanism ................

Fig. 3.2 The noise theory of Leeson-Cutler is based on the idea that the injected noise is multiplied by the transfer characteristic of the resonant network. Although this is an explanation for the $1 / f^{2}$ slope in the phase noise spectrum, it does not take the up-conversion of the injected $1 / f$ noise into account $\ldots .$.

Fig. 3.3 The response of an oscillator to an injected noise current can be modeled using two single-input, single-output systems, one for the excess phase and one for the instantaneous amplitude. . . . . . . . . . . . . . . . . .

Fig. 3.4 State diagram of a free running LC tank. The losses in the tank result in a decreasing oscillation. It is clear that the sensitivity to an injected noise pulse does not only depend on the moment of impact, but also on the amplitude . . . . . . .

Fig. 3.5 An injected signal with amplitude $I_{n}$ is converted to the spectrum of the excess phase $\phi(t)$. The signal is weighted by the Fourier coefficients of the ISF $\Gamma(x)$. Furthermore, a lowpass filtering is applied by the integral, in this way the signals close to the carrier or to one of the harmonics are dominant on the signals further away. The second step is the phase modulation of this excess phase. During this operation, the injected signal is up-converted to the carrier frequency (and to its harmonics) $\ldots \ldots \ldots \ldots \ldots \ldots \ldots \ldots$ 
Fig. 3.6 Similar to Fig. 3.5, this figure shows the translation of a noise spectrum towards the oscillator phase noise spectrum. During the convolution of the ISF and the injected noise, the noise contribution around each harmonic of the ISF is translated into noise around zero in the spectrum of the excess phase $\phi(t)$. Keep in mind, however, that the noise contributions appear around each of the ISF harmonics but are filtered by the integral operator. During the phase modulation step, the spectrum is up-converted towards the oscillator center frequency and the harmonics. Although the LTV theory of Hajimiri predicts an infinite noise power close to the carrier, a Lorentzian spectrum is drawn. . . . . . . . . . 73

Fig. 4.1 Typical block diagram of a harmonic (linear) oscillator . . . . . 93

Fig. 4.2 A parallel RLC network, as shown previously in Fig. 2.9a . . . 98

Fig. 4.3 Two typical structures of an integrated inductor. Above an electronic model is shown with some of the parasitics. Note that the number of components in the lumped inductor model needed to accurately simulate the behavior, depends on the wavelength of the applied signal. Due to its small dimensions (usually smaller than $1 \mathrm{~mm}$ ), and the lossy silicon substrate underneath, a lot of capacitive parasitics arise. Furthermore, the series resistance and Eddy currents in the substrate cause significant energy losses. . . . . . . . . . . . . . . . . . . . . . . . . 104

Fig. 4.4 Schematic drawing of the losses in an inductor, both caused by the flow of a changing current in the conductor. a Eddy currents are circular currents in the substrate, which cause a power loss and a decrease of the inductance. b Skin effect: due to circular currents in the conductor itself, the current is forced to the outer shell of the conductor . . . . . . . . . . . 105

Fig. 4.5 Comparison between different communication technologies: the bandwidth $B W$ is plotted versus the maximum

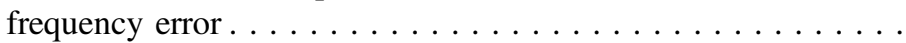

Fig. 4.6 Qualitative comparison of different properties of (uncompensated) crystal oscillators and silicon replacement circuits [176]. The dashed green line shows that a huge benefit of cost and size can be achieved when the oscillator can be integrated together with all other CMOS circuitry . . . . . . . .

Fig. 4.7 Block schematic of a generic harmonic oscillator. a A singleended amplifier and a feedback network. b Its differential equivalent. The amplifier compensates for the losses in the feedback network . . . . . . . . . . . . . . . . . 
Fig. 4.8 Typical differential implementation of an LC oscillator. On the left, the amplifier is implemented with NMOS transistors; on the right PMOS transistors are used. A combination of both is also possible [212] .

Fig. 4.9 A quadrature oscillator is forced towards its zero temperature frequency by cross-injection of quadrature signals [109]. The amplitude control is used to keep the amplifiers in the linear region. An external trimming circuit is used to trim $g_{m c}$ towards the $\mathrm{T}$-null frequency $\ldots \ldots \ldots \ldots \ldots \ldots$

Fig. 4.10 Low-noise differential Colpitts topology presented in [252].

Because of their better noise performance, only PMOS transistors are used $\ldots \ldots \ldots \ldots \ldots \ldots \ldots \ldots$

Fig. 4.11 Differential LC topology with an altered cross-coupling of the active devices $[117] \ldots \ldots \ldots \ldots \ldots \ldots \ldots$

Fig. 4.12 Tunable RC oscillator based on a single current

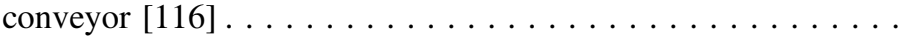

Fig. 4.13 Schematic drawing of a commonly used RC feedback network, called Wien bridge. On the right also its dual RL equivalent is shown. . . . . . . . . . . . . . . 120

Fig. 4.14 Transfer function of the Wien bridge feedback network . . . . . 120

Fig. 4.15 Schematic of the Wien bridge, which was used, similar to the Wheatstone bridge, to measure the value of capacitors in terms of frequency and resistance .............

Fig. 4.16 Schematic drawing of an astable multivibrator. On the right the output waveforms are shown. ............

Fig. 4.17 Schematic drawing of a relaxation oscillator based on a multivibrator [192]. On the right the output waveforms are shown. The reference voltage is equal to $1 \mathrm{~V}$ and the latch

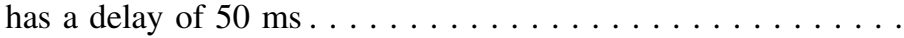

Fig. 4.18 The noise on the reference signal is directly translated to timing jitter on the next charging curve ...........

Fig. 4.19 Schematic of a low-jitter relaxation oscillator. The frequency is determined by $C_{2}, I_{1}$ and the value of $V_{c 2}$ at which the current through $M_{1}$ is equal to $I_{1}$, called $V_{\text {ref,2. }}$. On the right the output waveforms of a simulated Matlab model are shown. The output signal $V_{\text {out }}$ is obtained by putting $V_{c 1}$ through a comparator. As can be seen, $V_{\text {ref, }, 1}$ (dashed line) has no impact on the timing of the rising edges in the output

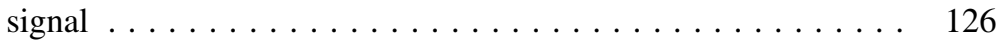

Fig. 4.20 Block diagram of a 3-stage ring oscillator . . . . . . . . . . . 127

Fig. 4.21 Schematic of a fully-differential delay stage with a symmetrical load as used in [244]. The delay can be controlled

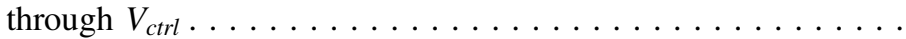


Fig. 4.22 Schematic of a mobility-based current source. The current $I_{0}$ is based on an externally applied reference voltage $V_{R} \ldots \ldots$.

Fig. 4.23 Schematic of a mobility-based current source. By controlling the inversion level of $M_{n 1}$, the output current $I_{B}$ can be made PTAT ......................

Fig. 4.24 Schematic drawing of an ETF. The heater $(\mathrm{H})$ is driven with a square wave: the heat transfers through the silicon to the temperature sensors $(\mathrm{S})$ which are laid out in circular pattern around the heater ..................

Fig. 4.25 Schematic drawing of an FLL using an ETF. The VCO locks on the thermal delay of the ETF, resulting in $\phi_{E T F}=\phi_{\text {ref }} \ldots$

Fig. 4.26 Qualitative comparison of different integrated oscillator topologies from literature $\ldots \ldots \ldots \ldots \ldots \ldots \ldots$

Fig. 5.1 Conventional Wien bridge topology using a passive feedback network and an opamp. Due to the feedback resistors, the amplifier has a gain of $3 \ldots \ldots \ldots \ldots \ldots \ldots \ldots \ldots \ldots \ldots$

Fig. 5.2 Schematic and small-signal model of a common-source amplifier with drain resistor $\ldots \ldots \ldots \ldots \ldots \ldots \ldots$

Fig. 5.3 Small-signal model of the improved Wien bridge oscillator Topology . . . . . . . . . . . . . . . . . . . . . . . . . . . 143

Fig. 5.4 Complete amplifier used in the Wien bridge oscillator . . . . . . . 144

Fig. 5.5 Gain-boosting amplifier for the lower cascode transistors . . . . 145

Fig. 5.6 Bode plot showing the gain of the gain-boosting amplifiers and the complete amplifier. The source impedance of the cascode transistors is also shown. The dashed lines indicate the active region of the gain boosters. . . . . . . . . . . 145

Fig. 5.7 Schematic of the amplitude-regulator circuit . . . . . . . . . . . 147

Fig. 5.8 Gain of the amplifier as a function of the voltage applied to the bridge transistor. . . . . . . . . . . . . . . . . 148

Fig. 5.9 Complete schematic of the Wien bridge oscillator. The gainboosting amplifiers and the amplitude-regulator circuit are omitted for clarity reasons . . . . . . . . . . . . . . . . 149

Fig. 5.10 Main noise sources in the transconductance amplifier . . . . . . 150

Fig. 5.11 Small-signal schematic of the differential oscillator. The noise contributions of the amplifier are grouped in $i_{n, A}^{2}$. Note that $v_{\text {out }}=v_{1}-v_{3} \ldots \ldots \ldots \ldots \ldots \ldots \ldots \ldots \ldots \ldots \ldots \ldots$

Fig. 5.12 Bode plot of the noise transfer functions. The sharp peak is the resonance frequency of the closed-loop system . . . . . . .

Fig. 5.13 Small-signal schematic of a single-ended Wien bridge oscillator. The propagation of the amplifier noise to the output is proven to be the same as in the differential case.

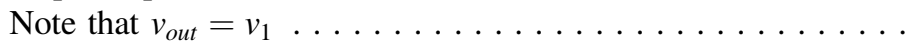


Fig. 5.14 ISF of the Wien bridge oscillator, normalized to the maximum output voltage $v_{\max }$. Two curves are shown, one for the input node and one for the output node of the feedback amplifier. In the lower graph, the output waveform of the oscillator is shown . . . . . . . . . . . . . .

Fig. 5.15 Chip photomicrograph of the implemented Wien bridge

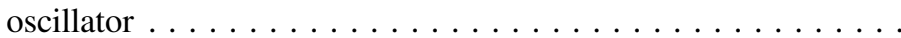

Fig. 5.16 Measured frequency deviation as a function of temperature for different samples. The frequency was normalized at $20{ }^{\circ} \mathrm{C}$; some samples did not work at $120{ }^{\circ} \mathrm{C} \ldots \ldots \ldots \ldots$

Fig. 5.17 Measured phase noise as a function of the carrier frequency offset for 3 different samples . . . . . . . . . . . . . . 156

Fig. 5.18 An LDO regulator typically consists of a voltage reference, an output transistor and a feedback amplifier . . . . . . . . .

Fig. 5.19 The used Wien bridge oscillator. The resistors in the feedback network are implemented by the output impedance of the amplifiers. $V_{v, \text { reg }}$ and $V_{i, \text { reg }}$ are biasing voltages, both delivered by the regulator circuitry. . . . . . . . .

Fig. 5.20 The two oscillators are coupled in opposite phase by the coupling capacitors $C_{c}$. Note that the current sources of the differentially oscillating amplifiers are shared to draw a constant current over time. The biasing voltage $V_{\text {rep }}$ is delivered by an amplifier replica; $V_{v, \text { reg }}$ and $V_{i, \text { reg }}$ are biasing voltages, both delivered by the regulator circuitry ........

Fig. 5.21 The voltage regulator is based on the $V_{t h}$-based voltage reference on the left. The output voltage is determined by the threshold voltages of $M_{1}$ and $M_{2} \ldots \ldots \ldots \ldots \ldots \ldots$

Fig. 5.22 Two regulators are used to provide a stable output voltage and current to the oscillator. The current regulator is directly connected to a replica of the amplifier used in the oscillator. The output current of the regulator only changes $0.02 \%$ over

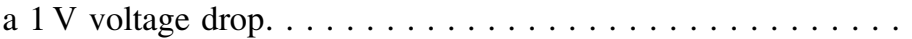

Fig. 5.23 Simulated output of the voltage and current regulator. The relative deviation compared to the output voltage at $1 \mathrm{~V}$ is shown. In the lower graph also the relative output frequency variation as a function of the supply voltage is shown . . . . .

Fig. 5.24 Simulated loop gain of the regulators $\left(T_{v, \text { reg }}\right.$ and $\left.T_{i, \text { reg }}\right)$ and the power supply rejection ratio (PSRR) at the output of the oscillator. The supply voltage is $1.0 \mathrm{~V}$. The current and voltage regulator have a phase margin of $70^{\circ}$ and $58^{\circ}$

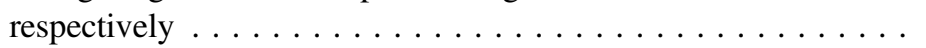


Fig. 5.25 Simulated output deviation of the regulators as a function of temperature. The upper graph shows the output voltage of the regulators. In the lower graph, the output current and the resulting oscillator frequency are shown. ...........

Fig. 5.26 Simulated output deviation of the regulators as a function of temperature when a resistor is used as the reference load of the current regulator. The upper graph shows the output voltage of the regulators and the voltage over the replica. In the lower graph, the output current through the oscillator replica and the resulting oscillator frequency are shown . . . . . 168

Fig. 5.27 Chip photomicrograph of the proposed voltage-independent oscillator; the active area measures $200 \mu \mathrm{m} \times 150 \mu \mathrm{m} \ldots \ldots$.

Fig. 5.28 The layout of the oscillator core is built completely pointsymmetrically. Every current is flowing in four directions to reduce the systematic influence of the silicon crystal and other non-isotropic influences..............

Fig. 5.29 Relative frequency deviation of the measured oscillator samples. On the right, the oscillation frequency of each sample at $1 \mathrm{~V}$ is reported. The maximum frequency variation is $104 \mathrm{ppm}$ over the $0.4-1.4 \mathrm{~V}$ supply voltage span . . . . . . .

Fig. 6.1 Block diagram of a pulsed-harmonic oscillator. Instead of a negative resistance or $g_{m}$, only an NMOS switch is used to drive the tank. PG is the pulse generator . . . . . . . . . .

Fig. 6.2 Simulated output waveform of a pulsed LC oscillator. Between pulses the amplitude is decaying due to the losses in the $\mathrm{LC} \operatorname{tank} \ldots \ldots \ldots \ldots \ldots \ldots \ldots \ldots \ldots \ldots$

Fig. 6.3 Some of the possibilities to build a tuned network. RC and LC networks are commonly used in fully integrated oscillator implementations. Crystals can only be used as an external

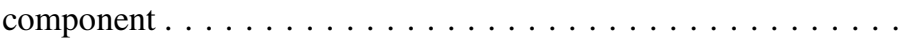

Fig. 6.4 A crystal can be modeled by a series LRC circuit with a capacitor in parallel [219]. This parallel capacitor $C_{p}$ is mainly caused by the parasitics of the casing . . . . . . . .

Fig. 6.5 When drawing the magnitude of a transfer function in the complex plane, it is seen that the Bode plot is the crosssection of this surface with the (positive) imaginary axis. Therefore, a Bode plot only shows the steady-state response

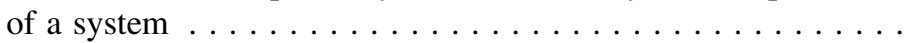

Fig. 6.6 Phasor diagram of the impulse response. To keep the period constant, a new Dirac impulse must be applied at the zero crossing of the cosine and not at the maximum of $h(t) \ldots \ldots$

Fig. 6.7 Schematic model of the tank. This model is numerically simulated in Matlab; the switch is closed (pulsed) once every $n$ cycles . . . . . . . . . . . . . . . . . . 
Fig. 6.8 Illustration of PW and MoI compared to the tank output

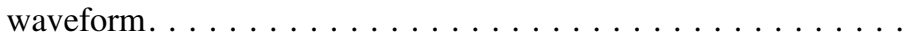

Fig. 6.9 Impact of a real pulse applied to the network of Fig. 6.7. When the right combination of pulse width (PW) and moment of impact $(\mathrm{MoI})$ are applied (indicated by the black line), the time between the zero crossings is not biased. All the values are normalized to half the LC tank oscillation period.

Fig. 6.10 Oscillation amplitude as a function of the pulse width (PW) and the moment of impact (MoI). A longer pulse leads to a higher amplitude, but also the moment of impact has a small influence on the output amplitude of the tank. All the values are normalized to half the LC tank oscillation period. . . . . . . .

Fig. 6.11 Optimal width $(\Delta T=0)$ of the applied pulses $(\mathrm{PW})$ as a function of the moment of impact (MoI) for different switch resistances $R_{p}$. Both axes are normalized to $T_{L C} / 2$, half the LC tank oscillation period . . . . . . . . . . . .

Fig. 6.12 Sensitivity of the oscillation period to the moment of impact. The derivative is calculated for the optimal PW-MoI combinations. All axes are normalized to half the oscillation period since this is the time span in which the pulse

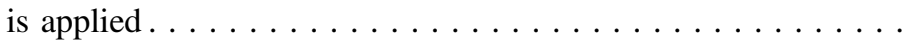

Fig. 6.13 Sensitivity of the oscillation period to the pulse width. The derivative is calculated for the optimal PW-MoI combinations. All axes are normalized to half the oscillation period since this is the time span in which the pulse is applied. . . . .

Fig. 6.14 Phase portrait of a pulsed oscillator. The LC network is similar to Fig. 6.7 with a switch discharging the state capacitor. An equal noise voltage $\Delta V$ is added at two different moments in the oscillation, causing a different phase shift $\Delta \theta$ in the output wave $\ldots \ldots \ldots \ldots \ldots \ldots$

Fig. 6.15 Top The ISF of the pulsed oscillator. The blue curve is the numerically simulated ISF; the red dashed curve represents the analytically calculated ISF during the free-running period. Both curves are almost identical. Bottom The corresponding output waveform of the LC tank. The red dashed line shows the pulses applied to the LC tank . . . . . . .

Fig. 6.16 Fast Fourier transform of the ISF. The most important frequency component is at $32 \cdot f_{0}=f_{L C} \ldots \ldots \ldots \ldots$

Fig. 6.17 The amplifier used to detect the LC output signal is a differential pair in combination with 2 differentially biased CMOS inverters. The differential pair is shown (right) together with the supply-independent biasing circuit

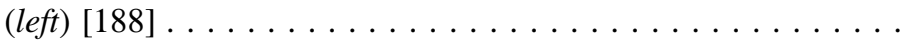


Fig. 6.18 Delay and peak-to-peak output signal of the amplifier for different input amplitudes at $V_{d d}=1.1 \mathrm{~V}$ and for a falling and a rising edge at the input. The circuit is able to detect a $10 \mathrm{mV}$ signal when the biasing current is $10 \mu \mathrm{A}$. When the current is decreased, the sensitivity also decreases........

Fig. 6.19 The ripple counter is built out of 5 modified TSPC

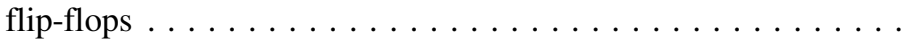

Fig. 6.20 The pulse width of the pulse generator is controlled by the inverter delay in combination with the output capacitor . . . . .

Fig. 6.21 Impact of the changing temperature on the output PW and MoI. The combined impact on the pulsed period

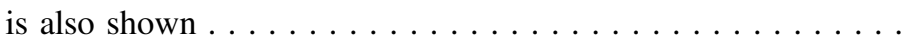

Fig. 6.22 Photomicrograph of a pulsed LC oscillator. Some other unrelated test circuitry is laid out under the inductor. The chip area is $1,750 \mu \mathrm{m}$ by $1,500 \mu \mathrm{m} . \ldots \ldots \ldots \ldots \ldots$

Fig. 6.23 Measured relative frequency error of the twelve measured samples. As predicted in Sect. 6.6.4.1, the frequency drops with increasing temperature $\ldots \ldots \ldots \ldots \ldots$

Fig. 6.24 Measured relative frequency error over different supply voltages of the twelve samples . . . . . . . . . . . . . . . . . 204

Fig. 6.25 Output jitter of the oscillator. The upper plot shows the simulated jitter, without supply noise. In the second plot, supply noise is added to simulate the noise injection of the digital circuitry as well as thermal noise to match the measured output jitter, which is shown in the third plot. The last plot shows the jitter on only the odd periods, since this is a more correct representation of the random (accumulated) noise in the system ..................

Fig. 7.1 System overview of the wireless sensor network (WSN) with RF clock distribution. The specifications of the clock carrier in terms of temperature and supply voltage stability are unimportant as long as the different network components can

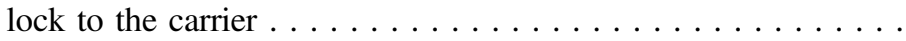

Fig. 7.2 Generic model of an oscillator, $H(s)$ is the tuned feedback network and $G(s)$ represents the amplifier. When a small current $i_{i n j}$ is injected, a phase shift is caused at the input of the feedback network. .................

Fig. 7.3 Amplitude and phase of the generic transfer function of the oscillator. The angular frequency is normalized to $\omega_{n}$ and the amplifier needs a gain of 4 to obtain a stable oscillation. A frequency shift causes a nonzero phase shift in the

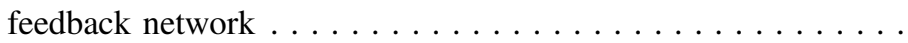


Fig. 7.4 Phasor diagram of the different currents in locked condition.

On the left, the oscillator current $i_{\text {osc }}$ leads the injected current $i_{i n j}$, introducing a positive phase shift $\left(\omega_{i}<\omega_{n}\right)$. On the right, $i_{\text {osc }}$ lags $i_{i n j}$, introducing a negative phase shift

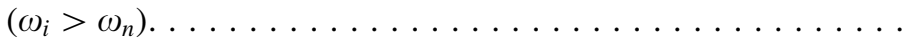

Fig. 7.5 Schematic of a relaxation oscillator using a Schmitt trigger. The current sources are switched on and off by the output signal of the Schmitt trigger. This results in a triangular output waveform $V_{C} \ldots \ldots \ldots \ldots \ldots \ldots \ldots$

Fig. 7.6 Waveforms in the relaxation oscillator. $V_{C}$ is the voltage on the capacitor while $I_{C}$ is the current from the current sources. All waveforms are scaled to be able to denote the necessary time parameters. . . . . . . . . . . . . . . . . . . . . . 214

Fig. 7.7 Pull-in time for an injection-locked oscillator as a function of the initial angle $\phi_{i, 0}$ and the steady state angle $\phi_{\infty}$. The time, normalized to $1 / \omega_{L}$, to enter the interval $\phi_{\infty}-0.01<$ $\phi_{i}(t)<\phi_{\infty}+0.01$ is shown since the actual pull-in process occurs exponentially $\ldots \ldots \ldots \ldots \ldots \ldots \ldots$

Fig. 7.8 Normalized beating frequency as a function of the normalized offset frequency. The influence of the injected signal is the largest when approaching the lock range. At the borders of the lock range, the beating frequency is equal to zero . . . . .

Fig. 7.9 Phase difference between the injected signal and the oscillator output when the injected signal is just out of the lock range $\left(\omega_{L}=1\right)$. The frequency difference between the injected signal and the oscillator output is also shown. When the phase shift is around $90^{\circ}\left(-90^{\circ}\right)$, the influence is the highest and the oscillator output 'tries to follow' the injected signal. From the moment the phase shift increases (decreases) further, the oscillator frequency falls back until the next clock edge arrives. The nominal oscillator frequency, $\omega_{n}$, is also shown in the graph $\ldots \ldots \ldots \ldots \ldots$

Fig. 7.10 One-port representation of an oscillator. The negative $G_{m}$ is slightly nonlinear to implement an amplitude control mechanism .................

Fig. 7.11 Block diagram of a first order PLL, used to model an injection-locked oscillator. . . . . . . . . . . . .

Fig. 7.12 Transfer characteristic for both the oscillator noise (top) and the noise on the injected signal (bottom). When the frequency difference $\Delta \omega_{n}$ between the natural oscillator frequency and the injected signal increases, the $-3 \mathrm{~dB}$ filter frequency

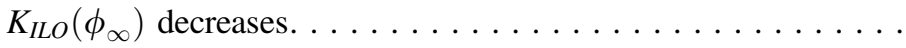


Fig. 7.13 Normalized $-3 \mathrm{~dB}$ frequency of the filter characteristic as a function of the normalized frequency difference between the natural oscillator signal and the injected signal $\Delta \omega_{n}$. The different curves are for different injection levels and are all normalized to their corresponding lock range $\omega_{L} \ldots \ldots \ldots \ldots$

Fig. 7.14 Example noise spectrum of an injection-locked oscillator as a function of the injected noise spectrum $S_{i n j}$ and the oscillator spectrum $S_{n n}$ for different values of $\phi_{\infty}$. The spectrum within the lock range is mainly determined by the injected spectrum and the oscillator noise at the edge of the lock range. The frequency is normalized to $\omega_{n}$ and $\omega_{L}=0.1 \ldots \ldots \ldots \ldots$

Fig. 7.15 Block diagram of the injection-locked oscillator system. After buffering the antenna signal, it is injected to the differential oscillator which acts as a 2-fold frequency divider. Also a phase detector is implemented to detect a lock condition or to further increase the lock range . . . . . . .

Fig. 7.16 Schematic of the two-stage RC feedback network, based on a Wien bridge oscillator . . ...............

Fig. 7.17 Schematic of the differential implementation of the proposed oscillator. By connecting the 2 oscillators differentially and by sharing the capacitor in the first stage, the oscillators are forced in opposite phase. The voltages $v_{s 1}$ and $v_{s 2}$ are used to implement a lock detection circuit and a circuit to increase the lock range. ....................

Fig. 7.18 The injection-locked oscillator can be described as a single balanced mixer. On the left the current with angular frequency $2 \cdot \omega_{i}$ is injected at the sources of the differential pair. This corresponds to the current source on the right with angular frequency $\omega_{i} . K_{m i x}$ is the conversion gain of the mixer . . . . . . . . . . . . . . . . .

Fig. 7.19 Average output frequency and phase difference as a function of the frequency of the injected signal. The phase curves can be obtained by mixing the oscillator signal (or a $90^{\circ}$-shifted version) with the injected signal . . . . . . . . . .

Fig. 7.20 Schematic of the integrating phase detector. The biasing of the detector is done by the differential stage on the right, making use of the common-mode signal coming from the oscillator biasing replica. The detector has two inputs: the antenna signal coming from the output of the first stage of the input amplifier and the common-mode source voltage of one of the two oscillator stages, $v_{s 1}$ or $v_{s 2}$. a Shows a single-ended version with a digital output; $\mathbf{b}$ is a differential implementation with a lower gain and an analog output. .... 
Fig. 7.21 Measured free-running frequency of 3 different samples. Also the minimum upper bound and maximum lower bound of the lock range of all 8 samples are shown as a function of a the temperature and $\mathbf{b}$ the supply voltage . . . . . . . 237

Fig. 7.22 Photomicrograph of the injection-locked oscillator. . . . . . . 238

Fig. 7.23 Block diagram of the clock and receiver circuitry. Only the input amplifier and the AM demodulator are working at the (high) carrier frequency .................

Fig. 7.24 System overview of the wireless sensor network (WSN) with RF clock distribution and coordination receiver. The specifications of the clock carrier in terms of temperature and supply voltage stability are unimportant as long as the different network components can lock to the wirelessly distributed clock signal. The implemented downlink makes network coordination possible and avoids data collisions . . . . .

Fig. 7.25 Schematic of the differential implementation of the proposed oscillator. By connecting the 2 oscillators in series, both transistor branches are oscillating differentially . . . . . . . .

Fig. 7.26 Schematic of the first and third stage of the four-stage input amplifier. Each stage has its own common-mode feedback, implemented by the two NMOS transistors on top. The first stage also has two biasing resistors to set the DC level of the antenna inputs. The input is AC-coupled and the third stage contains a DC-suppression capacitor . . . . . . . . .

Fig. 7.27 Bode plot of the 4-stage front-end amplifier stages. The gain of the first stage at $2.4 \mathrm{GHz}$ is around $15 \mathrm{~dB}$ or 6 . The total gain is between 6 and 720 , depending on the number

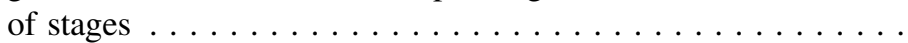

Fig. 7.28 AM-detector with an NMOS input pair. In practice, two AM-detectors, NMOS and PMOS, are used to generate a differential signal which can be injected differentially into

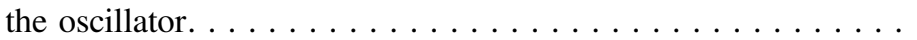

Fig. 7.29 Conversion gain of the envelope detectors for different input levels. On the left, the conversion gain is shown as a function of the input amplitude with $30 \%$ modulation depth. Note that this is the simulated output amplitude divided by the carrier amplitude and not the amplitude of the envelope. On the right, the same graph is shown for a signal with $100 \%$ modulation depth .................

Fig. 7.30 The output of both AM detectors is combined in a differential amplifier before injecting it into the oscillator. The gain of the amplifier can be controlled by adapting $V_{c t r l}$. The AM signal is also amplified by a two-stage amplifier to obtain a digital signal at the input of the phase detector. . . . . 
Fig. 7.31 Bode plot of the two baseband amplifiers including the highpass filter at the input. The first amplifier, which amplifies the baseband signal towards the oscillator, has a controllable gain. This is shown in the graph on the right (simulated for a $1 \mathrm{~V}$ supply voltage, at $30 \mathrm{MHz}) \ldots \ldots \ldots \ldots \ldots$

Fig. 7.32 Detailed overview of the receiver chain. The operations performed on the input spectrum are schematically drawn at the bottom. The combination of two phase detectors detects the negative envelope as well as the positive envelope of the input waveform, which results in a signal gain of $2 \ldots$.

Fig. 7.33 The hockey stick curves show the input referred noise of the $\mathrm{AM}$ receiver chain for different input resistances and a $30 \%$ modulation depth. The crossing of these curves with the $P_{\text {in }}-S N R_{\text {min }}$ curve determines the minimum detectable input signal level. On the left the input gain is equal to 6 (1 stage), on the right an input gain of 30 is assumed ( 2 stages)......

Fig. 7.34 The hockey stick curves show the input referred noise of the AM receiver chain for different input resistances and a $100 \%$ modulation depth. The crossing of these curves with the $P_{\text {in }}-S N R_{\text {min }}$ curve determines the minimum detectable input signal level. On the left the input gain is equal to 6 (1 stage), on the right an input gain of 30 is assumed ( 2 stages)......

Fig. 7.35 The receiver consists of a simple shift register of which the first flipflop is used as a phase detector. When a phase shift is applied to the input, the data register will clock. Depending on the most significant bit of the counter, which counts the clock cycles between two phase shifts, a zero or a one is received. In the subsequent clock cycle, the counter is reset . . .

Fig. 7.36 The simulated waveforms of an injection-locked receiver. Three times a $180^{\circ}$ phase shift is applied to the injected signal. The bottom graph shows the evolution of the phase difference. It takes around 10 periods until the output of the phase detector recovers from the phase shift. Note that the oscillator only has a weak nonlinearity in its amplifier, which results in a slow amplitude regulation $\ldots \ldots \ldots \ldots \ldots$

Fig. 7.37 Photomicrograph of the $40 \mathrm{~nm}$ injection-locked oscillator

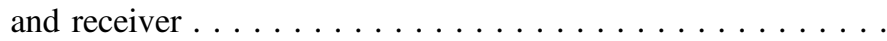

Fig. 7.38 Measured free-running frequency of 3 different samples of the $40 \mathrm{~nm}$ implementation. Also the estimated lock range is shown as a function of $\mathbf{a}$ temperature and $\mathbf{b}$ supply voltage . . .

Fig. 7.39 Operating range of the injection-locked receiver and the upper and lower limit of the lock range without controlling the baseband gain. On the left an input gain of 6 is used, on the right the input gain is equal to $30 \ldots \ldots \ldots \ldots$ 
Fig. 7.40 Operating range of the injection-locked receiver and the upper and lower limit of the lock range with baseband gain control. $V_{c t r l}$ was set to $0.75 \mathrm{~V}$ (or $V_{d d}-0.25 \mathrm{~V}$ ). On the left an input gain of 6 is used, on the right the input gain is equal

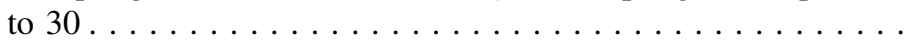

Fig. 8.1 Block diagram of a PLL-based sensor interface. The resistors $R_{s}$ represent the sensor input, which is connected to an oscillator. Due to the phase detector and the digitally controlled feedback resistors $R_{v}$, both oscillator frequencies are matched . . . . . . . . . . . . . . . . . . . 258

Fig. 8.2 Block diagram of the PWM-based sensor interface. Half of the oscillator stages are slowed down by the sensor signal, the other half is sped up. The output latch converts the oscillator signals into a PWM signal of which the duty cycle is proportional to the sensor value. . . . . . . . . . .

Fig. 8.3 Schematic of two oscillator stages and their interconnection. When a rising edge is applied to the input, the capacitor charges linearly and activates the next stage. The oscillator stage is reset by the output signal of stage $n+3$. In this way, all internal control signals are generated by the oscillator

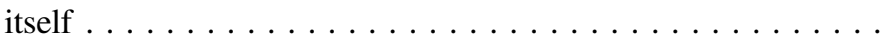

Fig. 8.4 Sketch of the output waveforms of subsequent oscillator stages. When using a CMOS differential pair, the switching delay when applying a linearly increasing input is exactly the same as in the case of an amplifier with an infinite gain. This is due to the point-symmetry of the differential pair . . . . . . .

Fig. 8.5 Transfer characteristic of a differential oscillator stage. The characteristic is point-symmetric around $V_{d}=0$. The linearized characteristic is shown as a dashed line. . . . . . . .

Fig. 8.6 Two configurations of a Wheatstone bridge: one with a current source on top, the other connected to an input voltage source ......................

Fig. 8.7 The sampling of the oscillator output signal when the duty cycle is equal to $1 / 3$. If only the black samples are taken into account, the samples need to be averaged over several periods. However, when the sampling speed is higher (all samples), only the first period is enough to obtain the right duty cycle. This shows the trade-off between the sampling speed and the bandwidth ...............

Fig. 8.8 Simplified schematic of the different noise contributions: a noise in the reference voltage $V_{S}$; b noise in the differential pair; c noise in the current source ............. 
Fig. 8.9 The jitter on the complete oscillator period $\left(T_{l, 0}+T_{h, 0}\right)$ as well as the jitter on half the oscillation period $\left(T_{l, 0}\right.$ or $\left.T_{h, 0}\right)$ are present in the interface's output signal . . . . . . . . . . . 273

Fig. 8.10 Normalized jitter (compared to the noise for a zero-input signal) of the output duty cycle as a function of the varying sensor input. The solid line uses a differential sensor; the dashed line is for a single-ended sensor. The noise sources themselves are constant; the variation is caused by the input-output transfer characteristic of the noise. . . . . . . . .

Fig. 8.11 The normalized jitter (compared to the noise for a zero-input signal) of the output duty cycle as a function of the varying sensor input for the noise generated by the current source. The variance of the noise increases linearly with the stage delay. The solid line uses a differential sensor; the dashed line is for a single-ended sensor . . . . . . . . . . . . . 276

Fig. 8.12 The most important design parameters of the PWM-based sensor interface in $130 \mathrm{~nm}$ CMOS ..............

Fig. 8.13 Output waveform of 4 oscillator stages. The linear charging curve as well as the slow switching and the point-symmetry of the switching operation are clearly visible . . . . . . . . . 280

Fig. 8.14 Linearity error of the output duty cycle as a function of the input voltage. In a the supply voltage $V_{d d}$ is varied from 0.9 to $1.6 \mathrm{~V}$; in $\mathbf{b}$ the temperature is varied from -40 to $120^{\circ} \mathrm{C}$

Fig. 8.15 Simulated error on the output duty cycle as a function of the input resistance. In a $V_{d d}$ is varied and the error is relative to the output value at $1.2 \mathrm{~V}$. In $\mathbf{b}$ the temperature is varied $(-40$ to $120^{\circ} \mathrm{C}$ ), the error values are relative to the output value at $30^{\circ} \mathrm{C}$. The maximum error appears at low temperatures for high input values and is equal to $1.2 \% \ldots \ldots \ldots \ldots 28 \ldots \ldots$

Fig. 8.16 Output spectrum of the sensor interface in $130 \mathrm{~nm}$. The noise bandwidth is equal to $1 \mathrm{MHz}$, the $100 \mathrm{kHz}, 25 \%$ amplitude input signal is clearly visible . . . . . . . . . . . . . . . 282

Fig. 8.17 The SNR and SNDR as a function of the input amplitude. From the SNDR also the ENOB can be calculated. A maximum is reached for an input amplitude between $10 \%$

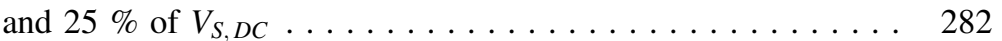

Fig. 8.18 The most important design parameters of the PWM-based sensor interface in $40 \mathrm{~nm}$ CMOS . . . . . . . . . . .

Fig. 8.19 Simulated output waveform of the $40 \mathrm{~nm}$ oscillator at a supply voltage of $1.2 \mathrm{~V}$. When the output of stage $7(n+3)$ is active, the current of stage $4(n)$ can be switched off . . . . . 
Fig. 8.20 Schematic of the current switch circuit. The current source is switched off when the stage is reset. When the oscillator is not oscillating, all current sources are switched on, which enables the startup of the oscillator . . . . . . . . . . .

Fig. 8.21 Linearity error of the output duty cycle as a function of the input sensor resistance. In figure a, the supply voltage $V_{d d}$ is varied from 0.6 to $1.6 \mathrm{~V}$. In figure $\mathbf{b}$ the temperature is varied from -40 to $120^{\circ} \mathrm{C}$

Fig. 8.22 Error of the output duty cycle as a function of the input sensor resistance. In a $V_{d d}$ is varied and the error is relative to the output value at $1 \mathrm{~V}$. At all supply voltages ( 0.9 to $1.6 \mathrm{~V})$ the error stays below $0.5 \%$. In $\mathbf{b}$ the temperature is varied $\left(-40\right.$ to $\left.120^{\circ} \mathrm{C}\right)$, the error values are relative to the output value at $70^{\circ} \mathrm{C}$. The maximum error appears at high temperatures for high input values and is around $1.1 \% \ldots \ldots$

Fig. 8.23 Linearity error of the output duty cycle as a function of the input sensor voltage. In figure a the supply voltage $V_{d d}$ is varied from 0.6 to $1.6 \mathrm{~V}$. In $\mathbf{b}$ the temperature is varied from

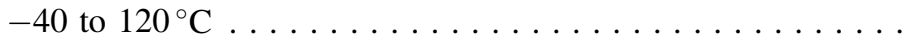

Fig. 8.24 Error on the output duty cycle as a function of the input sensor voltage. In a $V_{d d}$ is varied and the error is relative to the output value at $1 \mathrm{~V}$. At all supply voltages $(0.9$ to $1.6 \mathrm{~V})$ the error stays below $0.5 \%$. In $\mathbf{b}$ the temperature is varied $\left(-40\right.$ to $\left.120^{\circ} \mathrm{C}\right)$ and the error values are relative to the output value at $30^{\circ} \mathrm{C}$. The maximum error appears at high temperatures for high input values and is around $1.5 \% \ldots \ldots$

Fig. 8.25 The SNR and SNDR as a function of the input amplitude. From the SNDR also the ENOB can be calculated. The maximum in this case is reached at lower input amplitudes due to the increased nonlinearity . . . . . . . . . . . .

Fig. 8.26 The static nonlinearity of the sensor interface. In the upper graph, the nonlinearity is shown for a differential $\pm 180 \mathrm{mV}$ input signal. The graph below shows the nonlinearity for a smaller input range of $\pm 140 \mathrm{mV}$. The nonlinearity slightly decreases when the supply voltage increases. . . . . . . . . . 289

Fig. 8.27 Photomicrograph of the sensor interface in $40 \mathrm{~nm}$ CMOS. . . . 290

Fig. 8.28 Measured output spectrum of the sensor interface for a $1 \mathrm{~V}$ supply voltage and a $100 \mathrm{mV}$ differential signal. The SNDR determines the effective number of bits since it takes the noise as well as the distortion into account. The maximum FoM is reached for a $700 \mathrm{kHz}$ bandwidth . . . . . . . . .

Fig. 9.1 Block diagram of the wireless RFID tag. The power supply and clock input of different building blocks are controlled by the digital control logic. The state diagram of the finite state 
machine (FSM) is shown at the top. The length and content of the different states can be programmed by means of the shift register. . . . . . . . . . . . . . . . . . . 296

Fig. 9.2 Overview of the digital logic on the WSN tag. The FSM relies on different counters to make the right state transitions. The transmitted data, including the preamble, are scrambled using an LFSR before they are sent to the UWB transmitter. The shift register is used to control the length of the different states, the data and the LFSR scrambling code . . . . . . . .

Fig. 9.3 Profile of the energy consumption during one burst. In the initial phase, only the clock circuit and the receiver are switched on. When a correct tag-ID is received, the digital logic and the transmitter are switched on. Next the preamble, data and finally also the sensor data are subsequently transmitted. Afterwards, the tag is switched off. . . . . . . .

Fig. 9.4 Block diagram of the pulse generator and output stage with antenna. The capacitor $C$ is used to set the correct

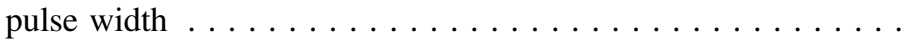

Fig. 9.5 Output waveform (left) and spectrum (right) of the UWB transmitter with a $250 \Omega$ load resistor. When using an appropriate antenna, the output spectrum is filtered and fits nicely within the FCC mask (dashed line). The design of the antenna is discussed in [209] . . . . . . . . . . . . .

Fig. 9.6 Received slow pulse train over a $50 \mathrm{~cm}$ wireless link. At higher distances, the received pulses disappear under the

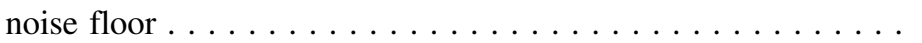

Fig. 9.7 The digital logic mainly consists of counters and shift registers. All of them are built using this basic flipflop . . . . . 301

Fig. 9.8 Block diagram of an $n$-bit programmable counter. The counter is set to the values in the shift register at the moment when PRG is set. . . . . . . . . . . . . . . . . 301

Fig. 9.9 Block diagram of 6-bit LFSR. The initial values as well as the used polynomial can be programmed through the shift register. Code lengths ranging from 1 to 63 bits can be generated using this hardware . . . . . . . . . . .

Fig. 9.10 Photograph of the automated multi-purpose measurement setup. The pcb on the right is a general-purpose PICcontroller platform with several human interface devices and a serial link to a PC or other measuring hardware. The board on the left contains all chip-specific hardware, going from DACs, to analog and digital buffers and digital potentiometers to steer the sensor interface. Both boards contain a controller, connected through an $\mathrm{I}^{2} \mathrm{C}$ link. The data shown on 
the display is steered by the left controller board to easily detect any communication errors between both controllers . . . 302

Fig. 9.11 Photograph of a bonded sample. a For the high-frequency signals mmcx connectors are used. b Photomicrograph of the wireless RFID tag . . . . . . . . . . . . . . . . . . . . . . 303

Fig. 9.12 The single-ended structure of the AM-detectors results in a high sensitivity to supply noise. Therefore, to improve the input sensitivity of the resulting tags, two connected dies have been used at measurement time. The supply coupling and sensitivity problem can be reduced by an improved detector topology, increased supply decoupling or the use of guard rings around the different blocks . . . . . . . . . . . . . 304

Fig. 10.1 Graphical comparison between the proposed oscillator designs and the state of the art. a Shows the power consumption as a function of the available voltage range. Both parameters contribute to the possible burst length. b Shows the temperature sensitivity in combination with the supply voltage sensitivity. Important to note are the highlyaccurate LC oscillators in the south-west corner, which have a high power consumption and need to be trimmed (the markers correspond to [3, 175, 177, 178, 234]). c Shows the noise as a function of the oscillator output frequency. It is clear that all implementations fall within the range of $\mathrm{RC}$ (IV-C) implementations . . . . . . . . . . . . . . . . . . . . . . 310

Fig. B.1 Typical block diagram of a harmonic (linear) oscillator (a). An oscillator with a linear feedback network and a nonlinear amplifier $(\mathbf{b}) \ldots \ldots \ldots \ldots \ldots \ldots \ldots \ldots \ldots \ldots \ldots \ldots \ldots \ldots \ldots$

Fig. B.2 Output voltage waveform and injected current for different transconductance amplifiers with soft distortion. The nonlinearity is necessary to control the amplitude but causes harmonics in the output waveform. The harmonics, on their turn, cause a small frequency drop.

a $Q=10, i(v)=1.5 \cdot v-0.1 \cdot v^{3}$. b $Q=10, i(v)=2.0 \cdot v-0.1 \cdot v^{3} \ldots \ldots \ldots \ldots \ldots$ 


\section{Tables}

Table 1.1 Summary of the target specifications ........... 25

Table 2.1 Commonly used PSD functions to characterize the frequency stability of an oscillator signal . . . . . . . 50

Table 3.1 Maximum limits of the phase noise FoM. . . . . . . . . . 89

Table 4.1 Comparison of some implementations from literature ..... 134

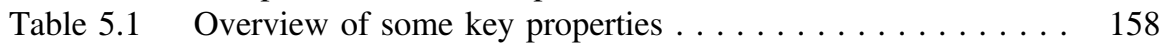

Table 5.2 Comparison to the state of the art for the temperature-independent oscillator. . . . . . . . . . . . . 159

Table 5.3 Comparison to the state of the art for the voltage-independent oscillator. . . . . . . . . . . . . 171

Table 6.1 Overview of the measured key properties (12 samples) . . . . 203

Table 6.2 Comparison of the oscillator to the state of the art ..... 206

Table 7.1 Overview of the key measurement properties (8 samples) . . 236

Table 7.2 Overview of the implemented prototype versions ....... 237

Table 7.3 Overview of the key measurement and simulation

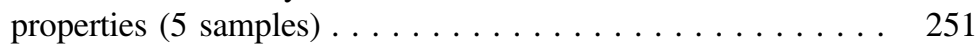

Table 7.4 Comparison of the injection-locked clock generators to the state of the art ................... 255

Table 7.5 Comparison of the receiver to the state of the art ...... 256

Table 8.1 The most important design parameters of the PWM-based sensor interface in $130 \mathrm{~nm}$ CMOS . . . . . . . . . . . 279

Table 8.2 The most important design parameters of the PWM-based sensor interface in $40 \mathrm{~nm}$ CMOS . . . . . . . . . . . 283

Table 8.3 Comparison of the key simulated properties

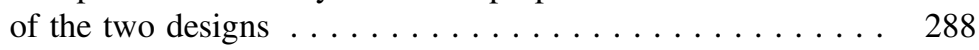

Table 8.4 Overview of the key properties (5 samples) . . . . . . . . 289

Table 8.5 Comparison to the state of the art in sensor interfaces . . . . . 291

Table 9.1 Comparison of the developed tag to the state of the art .... 305

Table 10.1 Comparison of the presented time references ......... 309

Table B.1 Coefficients of the different harmonics of the output waveform for the example ocillator............ 336 
Table C.1 The Allan variance for different slopes of the PSD . . . . . 346

Table C.2 Values of the different structure functions, corresponding to the different areas in the power-law noise spectrum. . . . . 348

Table C.3 $\tau$-dependency of structure functions of deterministic and stochastic processes. . . . . . . . . . . . . . 349

Table D.1 Comparison to the state of the art ........... 351

Table D.2 Comparison to the state of the art: noise ......... 353

Table D.3 Comparison to the state of the art: voltage dependency . . . . 354

Table D.4 Comparison to the state of the art: temperature dependency ...................... 356 